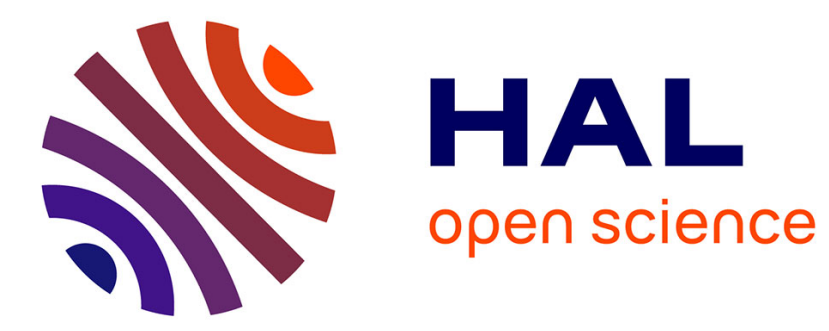

\title{
Lee waves from a sphere in a stratified flow
}

Bruno Voisin

\section{To cite this version:}

Bruno Voisin. Lee waves from a sphere in a stratified flow. Journal of Fluid Mechanics, 2007, 574, pp.273-315. 10.1017/S0022112006004095 . hal-00268807

\section{HAL Id: hal-00268807 https://hal.science/hal-00268807}

Submitted on 30 Mar 2011

HAL is a multi-disciplinary open access archive for the deposit and dissemination of scientific research documents, whether they are published or not. The documents may come from teaching and research institutions in France or abroad, or from public or private research centers.
L'archive ouverte pluridisciplinaire HAL, est destinée au dépôt et à la diffusion de documents scientifiques de niveau recherche, publiés ou non, émanant des établissements d'enseignement et de recherche français ou étrangers, des laboratoires publics ou privés. 


\title{
Lee waves from a sphere in a stratified flow
}

\author{
B. VOISIN \\ Laboratoire des Écoulements Géophysiques et Industriels, UJF, INPG, CNRS; BP 53, \\ 38041 Grenoble, France \\ Bruno.Voisin@hmg.inpg.fr
}

(Received 5 January 2005 and in revised form 14 September 2006)

Two asymptotic analyses of the generation of lee waves by horizontal flow at velocity $U$ of a stratified fluid of buoyancy frequency $N$ past a sphere of radius $a$ are presented, for either weak or strong stratification, corresponding to either large or small internal Froude number $F=U /(N a)$, respectively. For $F \gg 1$, the fluid separates into two regions radially: an inner region of scale $a$ with three-dimensional irrotational flow unaffected by the stratification, and an outer region of scale $U / N$ with small-amplitude lee waves generated by the $O(1)$ vertical motion in the inner region. For $F \ll 1$, the fluid separates into five layers vertically: from the lower dividing streamsurface situated at a distance $U / N$ above the bottom of the sphere to the upper dividing streamsurface situated at a distance $U / N$ below the top, there is a middle layer with two-dimensional horizontal irrotational flow; from the upper dividing streamsurface to the top of the sphere, and from the lower dividing streamsurface to the bottom, there are top and bottom transition layers, respectively, with three-dimensional flow; above the top and below the bottom, there are upper and lower layers, respectively, with small-amplitude lee waves generated by the $O(F)$ vertical motion in the transition layers.

The waves are calculated where they have small amplitudes. The forcing is represented by a source of mass: for $F \gg 1$, the surface distribution of singularities equivalent to the sphere in three-dimensional irrotational flow; for $F \ll 1$, the horizontal distribution of singularities equivalent, in the upper (resp. lower) layer, to the flat cut-off obstacle made up of the top (resp. bottom) portion of the sphere protruding above (resp. below) the upper (resp. lower) dividing streamsurface. The analysis is validated by comparison of the theoretical wave drag with existing experimental determinations. For $F \gg 1$, the drag coefficient decreases as $(\ln F+7 / 4-\gamma) /\left(4 F^{4}\right)$, with $\gamma$ the Euler constant; for $F \ll 1$, it increases as $(32 \sqrt{ } 2) /$ $(15 \pi) F^{3 / 2}$. The waves have the crescent shape of the three-dimensional lee waves from a dipole, modulated by interferences associated with the finite size of the forcing. For strong stratification, the hydrostatic approximation is seen to produce correct leading-order drag, but incorrect waves.

\section{Introduction}

The generation of internal gravity waves, called lee waves, by the horizontal motion of a body in a density-stratified fluid, or equivalently by the horizontal flow of a density-stratified fluid past an obstacle, is a problem of considerable geophysical importance; see e.g. Wurtele, Sharman \& Datta (1996). The present paper deals with its simplest form: the uniform horizontal motion of a body in an unbounded uniformly stratified Boussinesq fluid otherwise at rest, or equivalently the steady flow, otherwise horizontal and without shear, of a semi-infinite uniformly stratified Boussinesq fluid over an obstacle lying on a plane horizontal surface. 
This flow has been studied for over half a century. Early investigations, reviewed by Miles (1969), have focused on two-dimensional obstacles. Later, attention has turned gradually to three-dimensional obstacles, often considered within the hydrostatic approximation, reviewed by Smith $(1989 b)$. To date, however, the analytical description of the flow remains incomplete; in three dimensions, in particular, it is restricted to obstacles that are either small or flat, or to motion that is either hydrostatic or slow. We focus here on three-dimensional theory; for a comprehensive survey of theory, experiment and simulation for a wide range of obstacles, stratifications and incoming flows, the reader is referred to Baines (1995).

One of the most recent three-dimensional contributions is the analysis by Greenslade (2000) of the measurements by Mason (1977) and Lofquist \& Purtell (1984) of the drag on a sphere in a stratified flow. With $N$ the buoyancy frequency, $v$ the kinematic viscosity, $U$ the velocity of the flow and $a$ the radius of the sphere, the relevant similarity parameters are the internal Froude number $F=U /(\mathrm{Na})$ and the Reynolds number $R e=2 U a / v$.

Experimentally, the drag coefficient $C_{D}(R e, F)$ has been separated by Lofquist \& Purtell (1984) into two contributions: one, $C_{D}(R e, \infty)$, without the stratification, and the other, $\Delta C_{D}(R e, F)$, specific to it, so that

$$
C_{D}(R e, F)=C_{D}(R e, \infty)+\Delta C_{D}(R e, F) .
$$

For the ranges of values of $R e$ considered, both contributions turn out to be effectively independent of $R e$ : the former has been verified by Mason (1977), for $1600<R e<12000$, to vary only weakly, between 0.4 and 0.6 , with $R e$, as is normal when the wake of the sphere is turbulent (see e.g. Batchelor 1967, §5.11; Landau \& Lifshitz 1987, §45); and the latter has been verified by Lofquist \& Purtell, for $150<R e<5000$, to exhibit no effect of $R e$. Hence, to a good approximation, we may write

$$
C_{D}(F)=C_{D}(\infty)+\Delta C_{D}(F),
$$

with $C_{D}(\infty)$ a constant and $\Delta C_{D}(F)$ an inviscid stratified drag coefficient. At $R e=200$, the numerical study of Hanazaki (1988) has confirmed that friction plays a negligible role in $\Delta C_{D}(F)$ compared with pressure. Accordingly, viscosity will be neglected in the following.

Theoretically, the drag coefficient $C_{D}(F)$ has been separated by Greenslade (2000) into two contributions, $C_{D}^{\text {waves }}(F)$ associated with wave generation and $C_{D}^{\text {wake }}(F)$ with wake formation, so that

$$
C_{D}(F)=C_{D}^{\text {waves }}(F)+C_{D}^{\text {wake }}(F),
$$

implying

$$
\Delta C_{D}(F)=C_{D}^{\text {waves }}(F)+C_{D}^{\text {wake }}(F)-C_{D}(\infty) .
$$

Greenslade has plotted in this form the data of Mason (1977) and Lofquist \& Purtell (1984), and compared them with the output of two theories: for large $F$, the combination of a prediction of the wave drag by Gorodtsov \& Teodorovich (1982) and of the assumption of weak variations of the wake drag with $F$ as $F \rightarrow \infty$, yielding

$$
C_{D}^{\text {waves }}(F) \sim \frac{\ln F+7 / 4-\gamma}{4 F^{4}}, \quad C_{D}^{\text {wake }}(F) \sim C_{D}(\infty),
$$




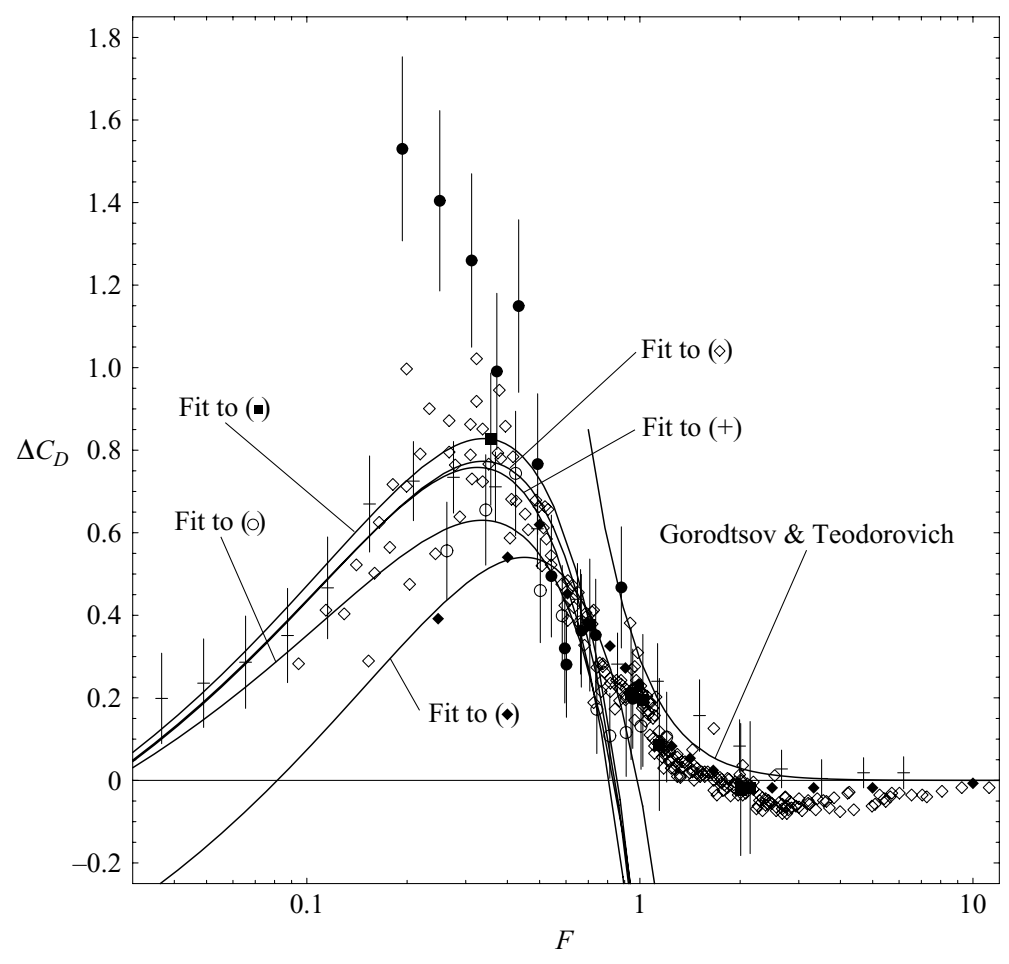

FIGURE 1. Experimental and numerical determinations of the stratified drag coefficient $\Delta C_{D}$ of a sphere, or plane-mounted hemisphere, as a function of the internal Froude number $F$ (adapted from Greenslade 2000, by kind permission of the author). The data points of Mason $(1977)(+)$, Lofquist \& Purtell (1984) $(\diamond)$, Hanazaki (1988) ( $\bullet)$, Shishkina (1996) (ם), Vosper et al. (1999) for the large hemisphere HS1 (๑) and the small hemisphere HS2 (O) are combined with continuous lines representing the models (1.5) for large $F$ and (1.6) for small $F$. The vertical error bars correspond to the standard errors plotted by Mason, to the error of 0.08 mentioned by Shishkina and to the accuracy of $10 \%$ mentioned by Vosper et al., in all three cases for $C_{D}(F)$ and $C_{D}(\infty)$ independently. The discrepancy at $F$ smaller than, say, 0.4 between the measurements for HS1 on one hand, and HS2 on the other hand, is attributed by Vosper (2000) to the finite width of the experimental tank used by Vosper et al:: at small $F$, the flow past the hemisphere is mostly horizontal and affected for HS1, due to its size, by the nearby sidewalls. This phenomenon, called blockage, must not be mistaken for that, called blocking, discussed in $\S 2.1$.

with $\gamma \approx 0.577$ the Euler constant; for small $F$, a combination of dimensional and geometrical considerations, yielding

$$
C_{D}^{\text {waves }}(F) \sim B F^{3 / 2}, \quad C_{D}^{\text {wake }}(F) \sim C F^{1 / 2}\left(1-\frac{5}{4} F\right),
$$

with $B$ and $C$ two constants obtained from statistical fit to the data. The comparison is reproduced in figure 1 , where the experimental data of Mason and Lofquist \& Purtell are complemented with those, experimental, of Shishkina (1996) and Vosper et al. (1999), and those, numerical, of Hanazaki (1988). The details of the fit are given in table 1. The data all appear consistent with one another and with the theory, except for Hanazaki, probably because of the low value of $R e$, and for the large hemisphere HS1 of Vosper et al., probably because of the small relative width of the experimental tank. 


Data set
Mason
Lofquist \& Purtell
Hanazaki
Shishkina
Vosper et al.

$$
\begin{gathered}
\text { Range of } R e \\
1600<R e<12000 \\
150<R e<5000 \\
\operatorname{Re}=200 \\
800<\operatorname{Re}<5000 \\
16000<\operatorname{Re}<78000
\end{gathered}
$$

\begin{tabular}{c}
\multicolumn{1}{c}{$B$} \\
$0.75 \pm 0.34$ \\
$0.87 \pm 0.20$ \\
$1.54 \pm 0.39$ \\
$0.93 \pm \infty$ \\
$0.71 \pm 0.50$
\end{tabular}

Points

$\begin{array}{lr}3.34 \pm 0.10 & 5 \\ 3.32 \pm 0.06 & 60 \\ 3.02 \pm 0.12 & 5 \\ 3.45 \pm \infty & 2 \\ 2.74 \pm 0.16 & 7\end{array}$

TABLE 1. Application of Greenslade's (2000) model (1.6) of the drag coefficient $C_{D}$ of a sphere at small $F$ to the experimental and numerical data of Mason (1977), Lofquist \& Purtell (1984), Hanazaki (1988), Shishkina (1996) and Vosper et al. (1999), represented in figure 1; for the last, only the small hemisphere HS2 has been taken into account. The constants $B$ and $C$ follow from a least-square fit of $\left[\Delta C_{D}(F)+C_{D}(\infty)\right] / F^{1 / 2}$ as a linear function of $F$ to each set of data within the range $0.2<F<0.8$. The values and associated errors are determined based on the estimates and standard errors of the fit, respectively; in each case the number of data points involved in the fit is indicated. For the constant $C_{D}(\infty)$, the values given by Mason and Hanazaki have been used directly; following Greenslade, the values omitted by Lofquist $\&$ Purtell and Shishkina have been replaced by that given by Mason; and the mean of the values, ranging between 0.32 and 0.50 , in figure $2(b)$ of Vosper et al. has been taken.

The present paper uses the sphere as a paradigm of three-dimensional obstacles, and Greenslade's (2000) analysis as a testbed for models of three-dimensional generation of lee waves. Two such models are proposed, appropriate at large and small $F$, respectively; they are applied to calculate both the drag and the waves. For the drag, only the wave contribution $C_{D}^{\text {waves }}$ is considered, denoted $C_{D}$ for short; it must, however, be kept in mind that, according to (1.6), the wake contribution $C_{D}^{\text {wake }}$ is dominant at small $F$. For the waves, only the lee waves generated by the displacement of the fluid by the sphere alone are considered, and not the so-called 'random' internal waves generated by its wake; it must, however, be kept in mind that, according to e.g. Bonneton, Chomaz \& Hopfinger (1993), the random waves are dominant at, say, $F \gtrsim 4$.

The paper is organized as follows. Section 2 reviews the existing theories of the generation of lee waves, first in two dimensions and then in three dimensions. Special attention is paid to re-organizing and systemizing all the approximations and their parameter regimes of validity. Based on the review, $\S 3$ relates, at large $F$, the representation of the sphere underlying (1.5a) to the asymptotic structure of the flow. At small $F$, a similar analysis of this structure, governed by the presence of dividing streamsurfaces, leads to a new and original representation of the sphere, in a regime for which no such representation was previously available.

Section 4 presents the equations of motion briefly. Based on them, $\S 5$ calculates the wave drag in each regime, applying Mellin-Barnes integration to the evaluation of the integrals involved (see e.g. Paris \& Kaminski 2001, ch. 5). As $F \rightarrow \infty$ the result (1.5a), given by Gorodtsov \& Teodorovich (1982) without explanation, is recovered and extended; as $F \rightarrow 0$ the result (1.6a) is recovered and the value of the constant $B$ is predicted analytically as $(32 \sqrt{ } 2) /(15 \pi) \approx 0.96$, to be compared with its determinations in table 1. In this way the two representations of the sphere are validated. Section 6 uses them for the calculation of the waves in the far field, by a generalization of the approach of Voisin (1994) for point sources. The results are applied to two particular values of $F, F=1$ deemed 'large' and $F=1 / 4$ deemed 'small', for illustration and with no implications, at this stage, for the domains of validity of the representations.

Section 7 evaluates the order of magnitude of the motion in each region of the fluid, at both large and small $F$. This legitimates in particular why, at small $F$, in 
contradistinction to conventional thinking, the linear approximation can be applied to the waves but not the hydrostatic approximation. Section 8 summarizes the theoretical results of the paper, discusses their relevance in view of the available experimental and numerical results, and outlines future directions of research for making the theory quantitative.

\section{Existing theories}

\subsection{Two-dimensional theories}

For flow at the velocity $U$ of a fluid of buoyancy frequency $N$ over a plane-mounted two-dimensional obstacle of height $h$ and length $2 \ell$, the appropriate similarity parameters are the three numbers

$$
F_{h}=F=\frac{U}{N h}, \quad \epsilon=\frac{h}{\ell}, \quad F_{\ell}=\epsilon F=\frac{U}{N \ell},
$$

only two of which are independent. The internal Froude number $F$ is the square root of the ratio of the kinetic energy, $\rho_{0} U^{2} / 2$ per unit volume, of the incoming flow to the potential energy, $\rho_{0} N^{2} h^{2} / 2$ per unit volume, gained by the fluid as it rises from the base to the summit of the obstacle, with $\rho_{0}$ the density at rest. This number represents, accordingly, an estimate of the ratio of inertial to buoyancy forces in the flow. The other parameters are the aspect ratio $\epsilon$, and the ratio $\epsilon F$ of the scale $U / N$ of buoyancy-induced motion to the horizontal scale $\ell$ of the obstacle. In this sense, $F_{\ell}=\epsilon F$ may be viewed as a horizontal internal Froude number, based on obstacle half-length, and $F_{h}=F$ as a vertical internal Froude number, based on obstacle height.

On the assumptions of steady motion and no modification of the incoming flow at infinity upstream, a linear equation, derived by Long (1953), governs the finiteamplitude motion of the fluid. In a system of coordinates $\left(x_{1}, z\right)$ relative to the obstacle, with the $x_{1}$-axis directed horizontally downstream and the $z$-axis vertically upwards, Long's equation is written

$$
\left(\frac{\partial^{2}}{\partial x_{1}^{2}}+\frac{\partial^{2}}{\partial z^{2}}+\frac{N^{2}}{U^{2}}\right) \zeta=0
$$

for the vertical displacement $\zeta\left(x_{1}, z\right)$. It is accompanied, for an obstacle of elevation $z=f\left(x_{1}\right)$ above the horizontal plane $z=0$, by the nonlinear boundary condition

$$
\zeta\left[x_{1}, f\left(x_{1}\right)\right]=f\left(x_{1}\right) .
$$

This boundary-value problem has been solved exactly in a few cases, including vertical barriers (Miles 1968; Janowitz 1973), and semi-circular (Kozhevnikov 1963, 1968; Miles \& Huppert 1968; Aksenov, Gorodtsov \& Sturova 1986), semi-elliptical (Huppert \& Miles 1969) and bell-shaped (Laprise \& Peltier 1989) obstacles. For the most part, however, analytical progress has relied on approximations (Miles \& Huppert 1969), associated with limiting values of $F_{h}, F_{\ell}$ and $\epsilon$ and summarized in figure 2; see also Miles (1969) and Baines (1995, §§5.2-3).

(i) For $F_{\ell} \rightarrow \infty$ with $\epsilon$ fixed, so that $F_{h} \rightarrow \infty$, a separation arises between the small scales $h$ and $\ell$ of the obstacle on one hand, and the large scale $U / N$ of the stratification on the other hand. Near the obstacle, that is in an inner region of scale $\ell$, the motion is unaffected by the stratification and hence irrotational. Far from the obstacle, that is in an outer region of scale $U / N$, the motion is made up of smallamplitude lee waves. Matching, as did Baines \& Grimshaw (1979) by the method of 


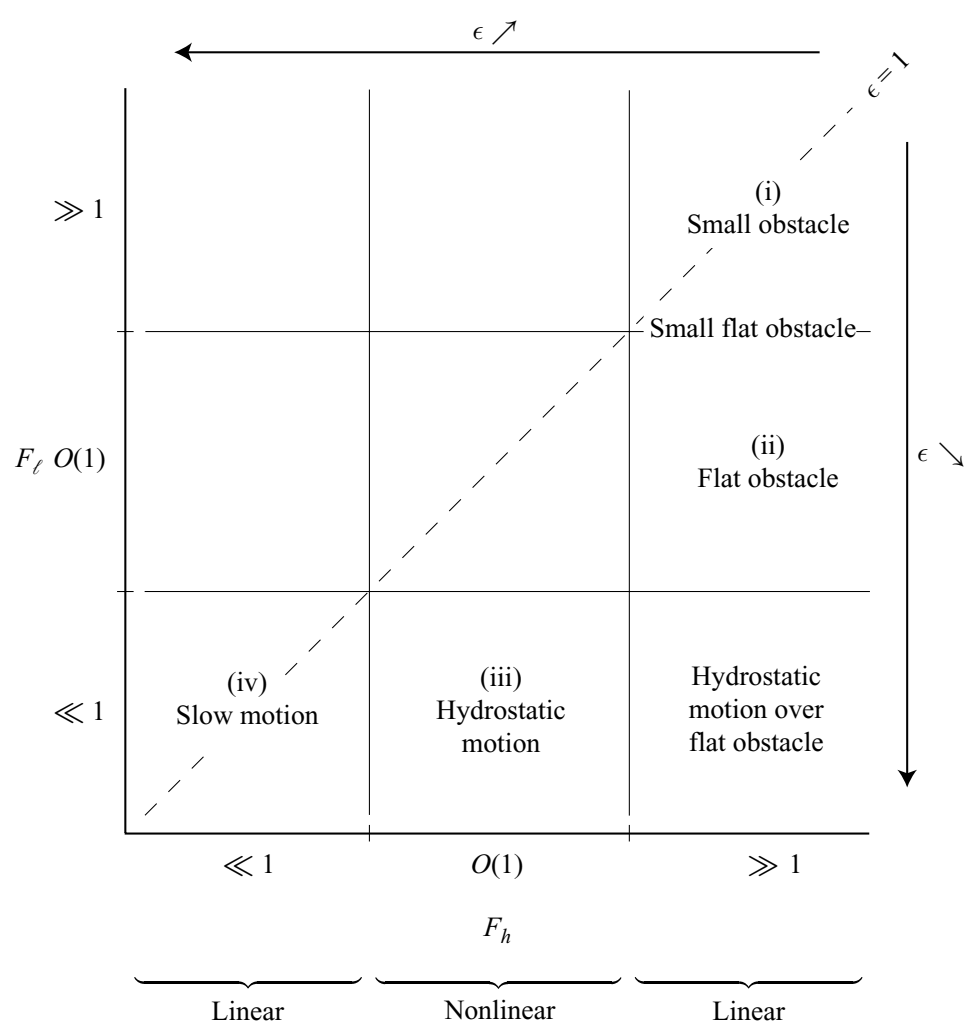

FIGURE 2. Approximations for two-dimensional flow at velocity $U$ of a stratified fluid of buoyancy frequency $N$ over a plane-mounted obstacle of height $h$ and half-length $\ell$, in terms of the vertical internal Froude number $F_{h}=U /(N h)$ and horizontal internal Froude number $F_{\ell}=U /(N \ell)$, with $\epsilon=h / \ell$ the aspect ratio (inspired by figure 2 of Baines \& Grimshaw 1979, considering cases (i) and (ii) and using coordinates $1 / F_{h}$ and $\epsilon$ ). For both $F_{h} \ll 1$ and $F_{h} \gg 1$ the problem is linear, though the linearization involves a different state of reference in each case.

matched asymptotic expansions, the two types of motion in an intermediate region $\ell \ll r_{1} \ll U / N$, with $r_{1}$ the distance from the obstacle, implies that the representation of the obstacle valid as $r_{1} \gg \ell$ in irrotational flow may be taken as the source term for the waves. With $A=\int f\left(x_{1}\right) \mathrm{d} x_{1}$ the area of the obstacle, $\rho_{0} A_{\star}$ its added mass (per unit length in the spanwise direction) in the absence of stratification and $\delta(x)$ the Dirac delta function, this representation is the dipole

$$
q\left(x_{1}, z\right)=2 U\left(A+A_{\star}\right) \frac{\partial}{\partial x_{1}} \delta\left(x_{1}\right) \delta(z),
$$

to appear in the equation of continuity as a source of mass releasing the volume $q$ of fluid per unit volume per unit time; see e.g. Batchelor (1967, § 6.4), Lighthill (1986, $\S 8.3)$ or Landau \& Lifshitz $(1987, \S 11)$. This approximation, that we shall call small obstacle, has also been called Rayleigh scattering in the literature, by analogy with the approximation of the same name in electrodynamics; see e.g. Jackson $(1999, \S 10.1)$ or Landau, Lifshitz \& Pitaevskii (1984, §92). The factor 2 in (2.4) and in similar formulae to follow is associated with the reflection at the rigid boundary $z=0$, and corresponds to the addition of the image of the obstacle through this boundary. 
(ii) For $\epsilon \rightarrow 0$ with $F_{\ell}$ fixed, so that $F_{h} \rightarrow \infty$, the obstacle $z=f\left(x_{1}\right)$ is a small perturbation to the plane horizontal surface $z=0$. The boundary condition (2.3) may be linearized, to become

$$
\zeta\left(x_{1}, 0\right)=f\left(x_{1}\right)
$$

which is equivalent to the representation of the obstacle as a singular source term

$$
q\left(x_{1}, z\right)=2 U \frac{\partial}{\partial x_{1}} f\left(x_{1}\right) \delta(z) .
$$

This approximation, that we shall call flat obstacle by analogy with the slender-body approximation of aeronautics and naval hydrodynamics (see e.g. Batchelor 1967, $\S 6.9)$, has also been called planar or linearized in the literature.

(iii) For $F_{\ell} \rightarrow 0$ with $F_{h}$ fixed, so that $\epsilon \rightarrow 0$, the streamwise variations of the motion occur on a scale $\ell$ long compared with that, $U / N$, associated with the stratification. The hydrostatic approximation $\partial / \partial x_{1} \ll N / U$ applies, simplifying Long's equation (2.2) to

$$
\left(\frac{\partial^{2}}{\partial z^{2}}+\frac{N^{2}}{U^{2}}\right) \zeta=0
$$

and turning the boundary condition (2.3) into an integral equation of Hilbert type, derived and solved independently by Miles \& Huppert (1969) and Lilly \& Klemp (1979).

All three approximations yield compatible results in their common areas of validity: for $h \ll \ell \ll U / N$, namely $1 \ll F_{\ell} \ll F_{h}$, the obstacle is both small and flat, and both source terms (2.4) and (2.6) reduce to $q\left(x_{1}, z\right)=2 U A \delta^{\prime}\left(x_{1}\right) \delta(z)$, since $A_{\star} \ll A$ for a flat obstacle; similarly, for $h \ll U / N \ll \ell$, namely $F_{\ell} \ll 1 \ll F_{h}$, the obstacle is flat and the motion hydrostatic, and the combination of the two simplifications (2.5) and (2.7) yields the linear hydrostatic solution of Drazin \& Su (1975).

However, both laboratory experiments and numerical simulations have revealed that the two basic assumptions of Long's model, namely steady motion and no upstream influence, become erroneous at sufficiently small $F_{h}<1$; see e.g. Baines $(1995, \S \S 5.1$ and 5.4).

Firstly, when the waves become so steep than the isopycnal lines, or lines of constant density, overturn, then breaking occurs and the motion ceases to be steady. To deal with this, Kantzios \& Akylas (1993) and Prasad, Ramirez \& Akylas (1996) have developed a generalization of Long's theory, appropriate for case (iii), based on the hydrostatic approximation but allowing weak unsteadiness and weak upstream influence. In this way, the realizability of Long's steady state has been assessed.

Secondly, fluid that has not enough kinetic energy to flow over the obstacle piles up in front of it, forming a region of stagnant fluid extending to infinity upstream; see e.g. Baines (1987). This phenomenon, known as upstream blocking, is attributable to the propagation upstream of internal waves, called columnar disturbances, of zero frequency and group velocity horizontal and larger than the incoming velocity; see e.g. Lighthill $(1978, \S 4.12)$. To deal with this situation, a fourth approximation has been introduced.

(iv) For $F_{h} \rightarrow 0$ with $\epsilon$ fixed, so that $F_{\ell} \rightarrow 0$, the incoming flow is slow and generates motion that is itself slow, allowing the linearization of the equations of motion around the state of rest of the fluid. For a semi-circular obstacle of radius $a=h=\ell$, at large time $t$, such that $N t \gg 1$, after impulsive start-up at time $t=0$, Bretherton (1967) and Krishna (1968) have obtained a superposition of two components: a steady horizontal 
blocked flow

$$
\begin{aligned}
& u_{1}=U \frac{z}{\left(z^{2}-a^{2}\right)^{1 / 2}}, \quad w=0 \quad(z>a), \\
& u_{1}=0, \quad w=0 \quad(0<z<a),
\end{aligned}
$$

where $\boldsymbol{u}_{1}=\left(u_{1}, w\right)$ is the velocity of the fluid, together with transient waves generated by the start-up. This approximation, that we shall call slow motion, fails in two respects: no steady lee waves are obtained, only transient internal waves and steady columnar disturbances; and the velocity field of these disturbances is singular at the level $z=a$ of the summit of the obstacle.

\subsection{Three-dimensional theories}

In three dimensions, none of the preceding finite-amplitude approaches holds any longer. As remarked by Drazin (1961), Yih (1967) and Zeytounian (1969), the same assumptions that led to Long's linear equation (2.2) in two dimensions lead to a nonlinear equation in three dimensions; see also Miles (1969) and Baines (1995, pp. 379-380). And the alternative nonlinear hydrostatic theory of Kantzios \& Akylas (1993) can only be generalized to steady motion over quasi-two-dimensional obstacles (Akylas \& Davis 2001), of much larger spanwise width than streamwise length. Hence, for fully three-dimensional obstacles, of comparable width and length, all existing approaches assume small amplitudes. With $\ell$ the horizontal scale of the obstacle and $h$ its vertical scale, two situations must be separated, as pioneered by Drazin (1961): weak stratification, corresponding to $F_{h} \gg 1$, and strong stratification, corresponding to $F_{h} \ll 1$.

For weak stratification, the assumption $F_{h} \gg 1$ allows, for the waves, the equations of motion to be linearized around the state of horizontal flow of the fluid at velocity $U$. With $y$ the spanwise coordinate, the resulting equation of propagation is

$$
\left[\frac{\partial^{2}}{\partial x_{1}^{2}}\left(\frac{\partial^{2}}{\partial x_{1}^{2}}+\frac{\partial^{2}}{\partial y^{2}}+\frac{\partial^{2}}{\partial z^{2}}\right)+\frac{N^{2}}{U^{2}}\left(\frac{\partial^{2}}{\partial x_{1}^{2}}+\frac{\partial^{2}}{\partial y^{2}}\right)\right] \zeta=0 .
$$

The boundary condition, for an obstacle of elevation $z=f\left(x_{1}, y\right)$ above the horizontal plane $z=0$, remains nonlinear and given by

$$
\zeta\left[x_{1}, y, f\left(x_{1}, y\right)\right]=f\left(x_{1}, y\right) .
$$

As in two dimensions, different approximations, associated with different ranges of values of $F_{\ell}$, have been developed for the solution of this boundary-value problem; compare with figure 2 .

For $F_{\ell} \gg 1$, approximation (i) is relevant: the obstacle is small and may be represented, in the outer region of scale $U / N$ where the waves are of small amplitude and governed by (2.9), by the dipole

$$
q\left(x_{1}, y, z\right)=2 U\left(V+V_{\star}\right) \frac{\partial}{\partial x_{1}} \delta\left(x_{1}\right) \delta(y) \delta(z),
$$

with $V=\iint f\left(x_{1}, y\right) \mathrm{d} x_{1} \mathrm{~d} y$ the volume of the obstacle and $\rho_{0} V_{\star}$ its added mass in the absence of stratification. This approximation has been used by Wurtele (1957), Crapper (1959), Wu (1965), Miles (1971), Sturova (1974, 1978), Dokuchaev \& Dolina (1977), Makarov \& Chashechkin (1981, 1982), Janowitz (1984) and Voisin (1994); see also Baines $(1995, \S 6.1 .3)$. 
For $F_{\ell}=O(1)$, approximation (ii) is relevant: the obstacle is flat and the boundary condition (2.10) may be linearized in the form

$$
\zeta\left(x_{1}, y, 0\right)=f\left(x_{1}, y\right)
$$

equivalent to the representation of the obstacle as a singular source term

$$
q\left(x_{1}, y, z\right)=2 U \frac{\partial}{\partial x_{1}} f\left(x_{1}, y\right) \delta(z)
$$

This approximation has been applied by Scorer (1956), Crapper $(1959,1962)$ and Umeki \& Kambe (1989) to various bell-shaped obstacles, and by Janowitz (1984) to an obstacle of arbitrary (flat) shape; see also Baines (1995, §6.1.3).

For $F_{\ell} \ll 1$, both approximations (ii) and (iii) are relevant: the obstacle is flat so that the boundary condition (2.10) simplifies to (2.12), and the motion is hydrostatic so that the equation of propagation (2.9) simplifies to

$$
\left[\frac{\partial^{2}}{\partial x_{1}^{2}} \frac{\partial^{2}}{\partial z^{2}}+\frac{N^{2}}{U^{2}}\left(\frac{\partial^{2}}{\partial x_{1}^{2}}+\frac{\partial^{2}}{\partial y^{2}}\right)\right] \zeta=0 .
$$

This linear hydrostatic approximation has been applied by Blumen \& McGregor (1976), Smith (1980) and Phillips (1984) to various bell-shaped obstacles; see also Baines (1995, $\S \S 6.1 .4-5)$. In order to enlarge its area of validity, Smith (1988) later reformulated the problem in isosteric coordinates, namely with the specific volume $\alpha$ as the vertical coordinate; see also Smith (1989b) and Baines (1995, §6.4). The surface $z=f\left(x_{1}, y\right)$ of the obstacle being, for large enough $F_{h}$, an isopleth of $\alpha$, the boundary condition (2.10) becomes exactly linear, without requiring the obstacle to be flat; one approximation remains, though, namely the small-amplitude long-wave approximation associated with the linear hydrostatic equation of propagation (2.14).

For strong stratification, the assumption $F_{h} \ll 1$ allows the equations of motion to be linearized around the state of rest of the fluid: the motion is slow, and approximation (iv) is relevant. For a hemispherical obstacle of radius $a=h=\ell$, at large time $t$, such that $N t \gg 1$, after impulsive start-up at time $t=0$, Grimshaw (1969) and Sarma \& Krishna (1972) have obtained a superposition of two components: a steady horizontal irrotational flow

$$
\begin{array}{lll}
\phi_{\mathrm{h}_{1}}=U x_{1}, & w=0 & (z>a), \\
\phi_{\mathrm{h}_{1}}=U x_{1}\left(1+\frac{a^{2}-z^{2}}{x_{1}^{2}+y^{2}}\right), & w=0 & (0<z<a),
\end{array}
$$

where $\boldsymbol{u}_{1}=\left(\partial \phi_{\mathrm{h}_{1}} / \partial x_{1}, \partial \phi_{\mathrm{h}_{1}} / \partial y, w\right)$ is the velocity of the fluid, together with transient waves generated by the start-up. The same approach has been applied by Vladimirov \& Il'in (1991) to semi-ellipsoidal obstacles. The first component, a columnar disturbance, is identical, for the hemisphere, to the leading-order solution of Drazin (1961) and Brighton (1978), obtained by looking for a steady solution of the nonlinear equations of motion as an expansion in powers of $F^{2}$; see also Baines (1995, § 6.7.1). Its nature, a horizontal flow, is associated with the fact that the fluid, not having enough kinetic energy to flow over the obstacle, flows around it.

It appears, however, that none of the preceding approaches is fully satisfactory: the small-obstacle approximation (i) has been noted by Miles (1971) and Janowitz (1984) to yield singular velocities along the $x_{1}$-axis downstream, and by Gorodtsov \& Teodorovich $(1980,1981)$ to yield infinite wave drag; neither the flat-obstacle approximation (ii) nor its combination with the hydrostatic approximation (iii) allows 
the consideration of bluff obstacles; and the slow-motion approximation (iv) has been noted by Drazin (1961) to produce a steady columnar disturbance singular at the level $z=h$ of the summit of the obstacle, and no steady lee waves.

\section{Representation of the forcing}

In order to address these issues, we consider a sphere of radius $a$ moving horizontally at the velocity $U$ in an unbounded stratified fluid of buoyancy frequency $N$. A system of coordinates $\left(x_{1}, y, z\right)$ relative to the sphere is used, with origin $O_{1}$ at its centre, the $x_{1}$-axis directed opposite to its motion and the $z$-axis vertically upwards. The position vector is $\boldsymbol{x}_{1}=\left(x_{1}, y, z\right)$, with $r_{1}=\left|\boldsymbol{x}_{1}\right|$ the radial distance. A subscript $\mathrm{h}$ indicates a horizontal projection, yielding $\boldsymbol{x}_{\mathrm{h}_{1}}=\left(x_{1}, y, 0\right)$ for the position and $r_{\mathrm{h}_{1}}=\left|\boldsymbol{x}_{\mathrm{h}_{1}}\right|$ for the distance.

The classical representation of a sphere as a source of lee waves, justified in theory for large internal Froude number $F=U /(N a)$, corresponding to region (i) of figure 2, but used in practice for any $F$, is the dipole (2.11) with the factor 2, corresponding to a plane-mounted obstacle, omitted. With $V=4 \pi a^{3} / 3$ the volume of the sphere and $V_{\star} / V=1 / 2$ its added mass coefficient in a homogeneous fluid (see e.g. Batchelor 1967, §6.8; Lighthill 1986, § 8.3; Landau \& Lifshitz 1987, §11), the dipole is written

$$
q\left(\boldsymbol{x}_{1}\right)=2 \pi a^{3} U \frac{\partial}{\partial x_{1}} \delta\left(x_{1}\right) \delta(y) \delta(z),
$$

and has the spectrum

$$
q(\boldsymbol{k})=2 \mathrm{i} \pi a^{3} U k,
$$

with $\boldsymbol{k}=(k, l, m)$ the wavenumber vector. Hereinafter, for any function $g$ of three space coordinates $x, y, z$ and one time variable $t$, Fourier transforms are defined by

$$
\begin{gathered}
g(\boldsymbol{k}, \omega)=\int g(\boldsymbol{x}, t) \exp [\mathrm{i}(\omega t-\boldsymbol{k} \cdot \boldsymbol{x})] \mathrm{d}^{3} x \mathrm{~d} t, \\
g(\boldsymbol{x}, t)=\frac{1}{(2 \pi)^{4}} \int g(\boldsymbol{k}, \omega) \exp [\mathrm{i}(\boldsymbol{k} \cdot \boldsymbol{x}-\omega t)] \mathrm{d}^{3} k \mathrm{~d} \omega,
\end{gathered}
$$

and similarly when fewer coordinates or variable are involved.

Unfortunately, the linear growth of the spectrum (3.2) with $k$ induces an algebraic divergence of the wave amplitude on the $x_{1}$-axis downstream (Miles 1971; Janowitz 1984), and a logarithmic divergence of the wave drag (Gorodtsov \& Teodorovich 1980, 1981). Clearly, a more elaborate representation is required. Based on visual examination of the flow, Makarov \& Chashechkin $(1981,1982)$ and Chashechkin (1989) have adopted a heuristic approach, modelling the sphere as a collection of sources and sinks, each with Gaussian spatial delocalization; in each particular case, the location, strength and size of the sources and sinks are fixed by comparison with experiment. We propose instead a predictive approach, based on asymptotic analysis of the structure of the flow. As anticipated by Drazin (1961), two types of stratification must be separated: weak stratification, corresponding to $F \gg 1$, and strong stratification, corresponding to $F \ll 1$.

\subsection{Weak stratification}

For $F \gg 1$, the representation of a moving body, of characteristic radius $a$, as a source of lee waves follows from the matching between an inner region of scale $a$, where the flow is to leading order irrotational and negligibly affected by the stratification, 


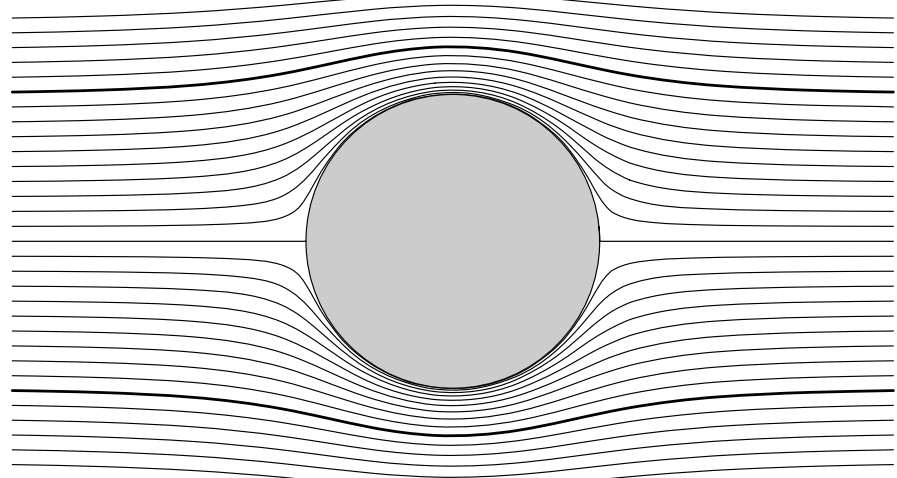

FIGURE 3. Streamlines for weakly stratified flow, at $F \gg 1$, past a sphere. The flow is represented to $O(1)$ in the inner region. At infinity upstream, the thick lines originate from the levels of the top and bottom of the sphere.

and an outer region of scale $U / N$, where lee waves propagate with small amplitudes. In two dimensions, this process has been suggested by Miles \& Huppert (1969) and implemented by Baines \& Grimshaw (1979); in three dimensions, it has been suggested by Miles (1971) and Murdock (1977) but remains to be implemented. All other investigations so far have considered either region in isolation.

The problem in the inner region has been formulated by Drazin (1961). To leading order, the flow is the $O(1)$ three-dimensional irrotational flow of a homogeneous fluid. For a sphere it is characterized, relative to the sphere, by the velocity potential

$$
\phi_{1}=U x_{1}\left(1+\frac{1}{2} \frac{a^{3}}{r_{1}^{3}}\right),
$$

the velocity $\boldsymbol{u}_{1}=\left(\partial / \partial x_{1}, \partial / \partial y, \partial / \partial z\right) \phi_{1}$ and the streamlines represented in figure 3 ; see e.g. Batchelor $(1967, \S \S 2.9$ and 6.8), Lighthill $(1986, \S 7.5)$ or Landau \& Lifshitz $(1987, \S 10)$. To next order, the stratification may have both buoyancy and inertial effects, so that the Boussinesq approximation is no longer valid; the buoyancy effect is $O\left(1 / F^{2}\right)$ and the inertial effect $O\left(N^{2} a / g\right)$, with $g$ the acceleration due to gravity. This next-order flow has been calculated by Hawthorne \& Martin (1955) for a sphere, taking both effects into account, by Murdock (1977) for a Rankine ovoid, retaining only the buoyancy effect, and by Eames \& Hunt (1997) and Palierne (1999) for a wider range of bodies, retaining only the inertial effect.

In the outer region, lee waves are observed. Physically, their source is the $O(1)$ vertical motion in the inner region. Mathematically, the representation of this source follows from matching, in an intermediate region $a \ll r_{1} \ll U / N$, to the inner region. To leading order, this procedure is usually thought to yield, as the source of the waves, the singularity that creates the far-field limit, as $r_{1} \gg a$, of the irrotational flow in the inner region (Miles 1971); namely the dipole (2.11), becoming (3.1) for the sphere (see e.g. Batchelor 1967, $\S 2.9$ and 6.4; Lighthill 1986, §§8.1-3; Landau \& Lifshitz 1987, §11).

To avoid the associated divergence, Gorodtsov \& Teodorovich (1982) have proposed using instead the distribution of singularities that creates exactly the same irrotational flow as the body in a homogeneous fluid; namely the distribution, located at the surface of the body and determined from the condition of fixed normal velocity at 
this surface, given for the sphere by

$$
q\left(x_{1}\right)=-\frac{3}{2} U \frac{x_{1}}{a} \delta\left(r_{1}-a\right)
$$

and having the spectrum

$$
q(\boldsymbol{k})=6 \mathrm{i} \pi a^{2} U \frac{k}{\kappa} j_{1}(\kappa a),
$$

with $\kappa=|\boldsymbol{k}|$ and where $j_{n}(x)$ and $J_{v}(x)$ are the spherical and cylindrical Bessel functions of the first kind, respectively, so that $j_{1}(x)=[\pi /(2 x)]^{1 / 2} J_{3 / 2}(x)=(\sin x) / x^{2}-(\cos x) / x$. We interpret this approximation as the use, as the source of the waves, of the distribution of singularities that creates the whole of the irrotational flow in the inner region, for all $r_{1} / a=O(1)$. At large wavelength, namely for $\kappa a \rightarrow 0$, this source has the same spectrum (3.2) as the dipole, thereby producing the same far-field inner flow; at small wavelength, namely for $\kappa a \rightarrow \infty$, the spectrum (3.6) vanishes, thereby avoiding the divergence of the outer waves. This is the model that we shall use for $F \gg 1$.

So far, the assumption of free slip at the surface of the body has been implicit. In the atmosphere, for flow over a topography of height $h$ comparable with the thickness of the viscous boundary layer at the ground, the assumption of no slip may be more appropriate; see e.g. Baines (1995, pp. 224-226). A similar separation takes place, for $F_{h} \gg 1$, between a lower layer with negligible effect of the stratification and an upper layer with small-amplitude lee waves. Bell-shaped topography has been considered in this way by Sykes (1978), for laminar flow, and by Hunt \& Richards (1984) and Hunt, Richards \& Brighton (1988), for turbulent flow.

\subsection{Strong stratification}

For $F \ll 1$, vertical motion is inhibited by the stratification. To leading order, the flow is $O(1)$ and purely horizontal, independent in each horizontal plane. Therefore, either above or below a moving body the fluid stays at rest, while at the level of the body a two-dimensional irrotational flow is observed, around each horizontal section of the body. For a sphere this flow has been given, relative to the sphere, by (2.15) in the half-space $z>0$, and is symmetrical with respect to the plane $z=0$. To next order, $O\left(F^{2}\right)$ vertical motion takes place, characterized for the sphere by the vertical displacement

$$
\begin{array}{ll}
\zeta=0 & (|z|>a), \\
\zeta=2 a F^{2} \frac{a z\left(x_{1}^{2}-y^{2}+z^{2}-a^{2}\right)}{\left(x_{1}^{2}+y^{2}\right)^{2}} & (|z|<a),
\end{array}
$$

and the isopycnal lines represented in figure 4.

Unfortunately, this model, introduced by Drazin (1961) and Brighton (1978), fails in two respects: first, at the levels of the top and bottom of the body, both the horizontal velocity and the vertical displacement diverge at the body and are discontinuous in the fluid; secondly, no steady lee waves are obtained, only steady columnar disturbances, together with, for impulsive start-up, transient waves calculated by Grimshaw (1969) and Sarma \& Krishna (1972) for a sphere.

Both failures are manifestations of the singular nature of the small- $F$ expansion: at small but finite $F$, as remarked by Drazin (1961), transition layers are formed in the fluid, with $O(F)$ thickness, at the levels of the top and bottom of the body; there, $O(F)$ vertical motion takes place, producing lee waves which propagate away into the fluid. Associated with the mathematical singularity is a physical reality, the existence 


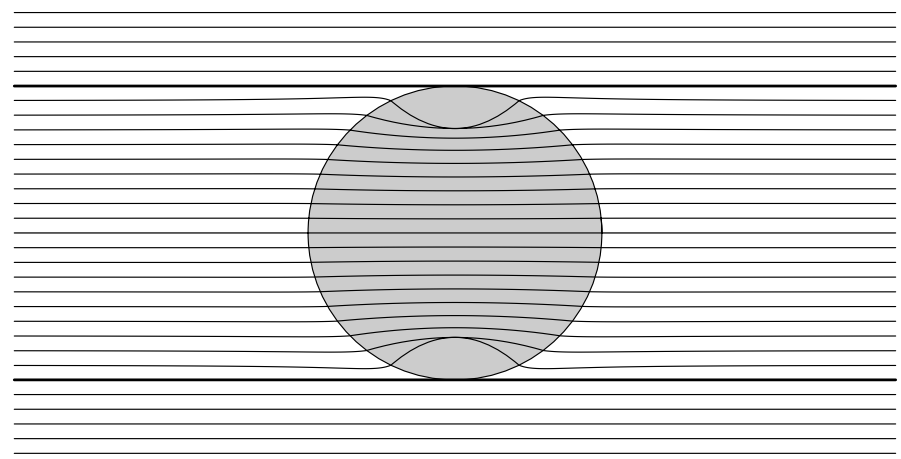

FiguRE 4. Isopycnals for Drazin's (1961) model of strongly stratified flow, at $F \ll 1$, past a sphere. The flow, which to $O(1)$ is purely horizontal, is represented to $O\left(F^{2}\right)$. The isopycnals are drawn in the vertical plane $y=0$ and on the sphere surface. At infinity upstream, the thick lines originate from the levels of the top and bottom of the sphere. For definiteness the value $F=0.1$ is used.

of the so-called dividing streamlines, more accurately streamsurfaces, each of which separates two layers of fluid: one, where the flow is horizontal and around the body, and the other, where the flow is three-dimensional and either over or under the body.

This concept, introduced by Sheppard (1956), is best presented for the flow at velocity $U$ over an obstacle of height $h$ and half-length $\ell$ lying on a plane horizontal surface $z=0$, with $F=U /(N h)$ the internal Froude number. The picture of Sheppard is quasi-static and considers only the conversion of the kinetic energy, $\rho_{0} U^{2} / 2$ per unit volume, of a fluid parcel in the incoming flow to the potential energy, $\rho_{0} N^{2} \zeta^{2} / 2$ per unit volume, gained by this parcel as it rises by a height $\zeta$. When $F<1$, only those parcels originating within a vertical distance $U / N$ below the summit are able to flow over the obstacle, the other parcels flowing around it. Accordingly, the fluid is divided into three horizontal layers: for $0<z<(1-F) h$, a lower layer with two-dimensional horizontal irrotational flow; for $z>h$, an upper layer where the incoming flow is unaffected by the presence of the obstacle; and in between, for $(1-F) h<z<h$, a summit layer, corresponding to Drazin's (1961) transition layer, with three-dimensional flow. The dividing streamsurface is identified as the plane separating the lower and summit layers, and the prediction $z=(1-F) h$ of its position is usually called Sheppard's criterion.

The extensive literature on strongly stratified flow has confirmed the qualitative value of this criterion; see e.g. Baines $(1995, \S \S 6.6$ and 6.7.3). Objections, of either theoretical (Smith 1989a), experimental (Baines \& Smith 1993) or numerical (Smith \& Grønås 1993) nature, have been raised to its quantitative value; they are associated with the dynamic role of the pressure, which the model neglects, a role that is increasingly large as $F$ increases. However, this quantitative value has been confirmed at $F \lesssim 0.1$ for bell-shaped obstacles by the experiments of Brighton (1978), Hunt \& Snyder (1980), Snyder et al. (1985) and Kadri et al. (1996), and by the numerical simulations of Smolarkiewicz \& Rotunno (1989, 1990), Suzuki \& Kuwahara (1992) and Miranda \& James (1992); at $F \lesssim 0.3$ for a triangular ridge of finite width by the experiments of Castro, Snyder \& Marsh (1983); at $F \lesssim 0.4$ for a sphere by the experiments of Sysoeva \& Chashechkin (1988), Chomaz et al. (1992), Lin et al. (1992), Chomaz, Bonneton \& Hopfinger (1993) and Vosper et al. (1999), and by the numerical simulations of Hanazaki (1988). It has also been verified in field experiments by Rowe et al. (1981), Ryan \& Lamb (1984), Egan (1984) and Spangler (1987). 
The construction of a singular mathematical model of the flow, building upon the regular model of Drazin (1961) and Brighton (1978) and accounting for the existence of the dividing streamsurface, has been initiated by Greenslade $(1992,1994)$ and Hunt et al. (1997). Lee waves are generated by the vertical motion in the summit layer and propagate, with small amplitudes, in the upper layer. In the lower layer, the flow, given for a hemisphere by $(2.15 b)$, is identical, for an obstacle of revolution of vertical axis and horizontal radius $b(z)$ at the level $z$, to that from a distribution of dipoles

$$
q\left(x_{1}\right)=2 \pi b^{2}(z) U \frac{\partial}{\partial x_{1}} \delta\left(x_{1}\right) \delta(y) H(h-z)
$$

located along the axis of the obstacle and of strength proportional to the area $\pi b^{2}(z)$ of its horizontal section; $H(x)$ denotes the Heaviside step function. Near the summit, the obstacle is assumed locally paraboloidal, with equation

$$
\frac{z}{h} \sim 1-\frac{\beta}{2} \frac{x_{1}^{2}+y^{2}}{\ell^{2}},
$$

where $\beta$ is a scaled curvature equal to 1 for a hemisphere, so that $b^{2}(z) \sim(2 / \beta) \ell^{2}(1-$ $z / h)$. The distribution becomes

$$
q\left(\boldsymbol{x}_{1}\right) \sim \frac{4 \pi}{\beta} \ell^{2} U\left(1-\frac{z}{h}\right) \frac{\partial}{\partial x_{1}} \delta\left(x_{1}\right) \delta(y) H(h-z) .
$$

Greenslade (1992) has proposed using this limiting form as the source of the waves, 'purely as an illustrative example' and with 'no guarantee that the result is accurate for the given [obstacle] profile'. On heuristic grounds, Newley, Pearson \& Hunt (1991) have adopted a similar representation, with Lorentzian horizontal delocalization, as $\left[1+\left(x_{1}^{2}+y^{2}\right) / \ell^{2}\right]^{-3 / 2}$.

Both representations fail to account for the fact that the waves are generated in the summit layer. Instead, forcing is considered to take place along the whole height of the obstacle, even becoming zero at the level of the summit in (3.10). More precisely, given that (3.10) is a limiting form of the source (3.8) of the $O(1)$ horizontal flow and $O\left(F^{2}\right)$ vertical motion in the lower layer, then its use for the waves amounts to taking this $O\left(F^{2}\right)$ motion as the forcing, given by $(3.7 b)$ for a hemisphere, instead of the $O(F)$ vertical motion in the summit layer.

Like Hunt \& Richards (1984), Hunt et al. (1988) and Greenslade (2000), we will adopt the alternative view that, the flow being, to leading order, purely horizontal in the lower layer and partly vertical in the summit layer, the two layers being separated by the dividing streamsurface, then the portion of the obstacle protruding above this surface in the summit layer acts as a cut-off obstacle, radiating lee waves in the upper layer while the lower layer acts as an absorber, preventing reflection at the rigid boundary $z=0$.

The cut-off obstacle, of height $h^{\prime}=h F$, half-length $\ell^{\prime} \sim \ell(2 F / \beta)^{1 / 2}$ and aspect ratio $\epsilon^{\prime} \sim \epsilon(\beta F / 2)^{1 / 2}$, is generally flat. (The case of a peaked original obstacle, of aspect ratio $\epsilon$ so large that $\epsilon^{\prime}=O(1)$, will be excluded in the following.) The flow over it is characterized by the horizontal internal Froude number $F_{\ell^{\prime}}=\epsilon(\beta F / 2)^{1 / 2} \ll 1$ and the vertical internal Froude number $F_{h^{\prime}}=1$. For such a flow, corresponding to the hydrostatic region (iii) of figure 2, it is usually thought that neither the boundary condition nor the equations of motion can be linearized, as the linearization, corresponding to the flat-obstacle approximation (ii), would require $F_{\ell^{\prime}}=O(1)$ and $F_{h^{\prime}} \gg 1$; see e.g. Baines (1995, p. 239). Accordingly, the approach has not been carried further in the literature. 


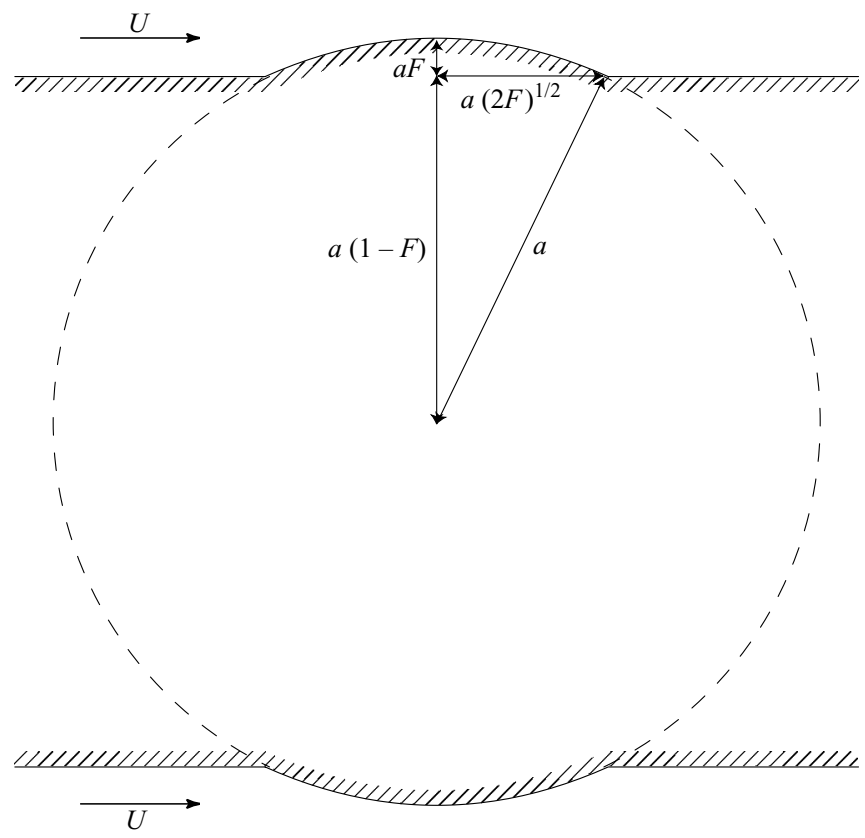

FiguRE 5. Forcing for strongly stratified flow, at $F \ll 1$, past a sphere. Only the top and bottom portions, of height $h^{\prime}=U / N=a F$ and half-length $\ell^{\prime}=a\left[1-(1-F)^{2}\right]^{1 / 2} \sim a(2 F)^{1 / 2}$, of the sphere contribute to wave radiation.

We argue, and will verify later in $\S 7.1$, that these reservations do not apply: for the waves in the upper layer, both the linear equation of propagation (2.9) and the linear boundary condition (2.12) can be used. The cut-off obstacle has the elevation

$$
f\left(x_{1}, y\right)=h\left(F-\frac{\beta}{2} \frac{r_{\mathrm{h}_{1}}^{2}}{\ell^{2}}\right) H\left[\ell\left(\frac{2 F}{\beta}\right)^{1 / 2}-r_{\mathrm{h}_{1}}\right] \text {, }
$$

above the horizontal plane $z=(1-F) h$ indistinguishable, to this order, from the plane $z=h$. Hence, by (2.13), it may be represented as the distribution of singularities

$$
q\left(x_{1}\right)=-\beta \epsilon U \frac{x_{1}}{\ell} H\left[\ell\left(\frac{2 F}{\beta}\right)^{1 / 2}-r_{\mathrm{h}_{1}}\right] \delta(z-h),
$$

of spectrum

$$
q(\boldsymbol{k})=4 \mathrm{i} \pi F h \ell U \frac{k}{\kappa_{\mathrm{h}}} \frac{J_{2}\left[\kappa_{\mathrm{h}} \ell(2 F / \beta)^{1 / 2}\right]}{\kappa_{\mathrm{h}} \ell} \exp (-\mathrm{i} m h),
$$

with $\boldsymbol{k}_{\mathrm{h}}=(k, l, 0)$ and $\kappa_{\mathrm{h}}=\left|\boldsymbol{k}_{\mathrm{h}}\right|$; the factor 2 associated in (2.13) with the reflection at the horizontal surface $z=0$ has been omitted, consistently with the interpretation of the lower layer as an absorber. In this way a new and original representation of a plane-mounted obstacle as a source of lee waves has been derived, appropriate for $F \ll 1$.

Returning to the moving sphere, the situation is, to leading order, as depicted in figure 5: the top portion of the sphere in the top layer $(1-F) a<z<a$, and its bottom portion in the bottom layer $-a<z<-(1-F) a$, act as cut-off flat bodies, radiating small-amplitude lee waves in the upper layer $z>a$ and lower layer $z<-a$, 
respectively; in between, purely horizontal flow takes place in the middle layer $|z|<(1-F) a$ which acts as an absorber isolating the waves radiated in the upper layer from those radiated in the lower layer. Each cut-off body can be represented by the distribution of singularities

$$
q_{ \pm}\left(\boldsymbol{x}_{1}\right)=-U \frac{x_{1}}{a} H\left[a(2 F)^{1 / 2}-r_{\mathrm{h}_{1}}\right] \delta(z \mp a)
$$

of spectrum

$$
q_{ \pm}(\boldsymbol{k})=4 \mathrm{i} \pi F a^{2} U \frac{k}{\kappa_{\mathrm{h}}} \frac{J_{2}\left[\kappa_{\mathrm{h}} a(2 F)^{1 / 2}\right]}{\kappa_{\mathrm{h}} a} \exp (\mp \mathrm{i} m a) .
$$

This is the model that we shall use for $F \ll 1$.

In the same way as the representation (3.5) of the sphere amounts to considering, for $F \gg 1$, the $O(1)$ flow illustrated in figure 3 as the basic flow responsible for the generation of lee waves, the representation (3.14) amounts to considering, for $F \ll 1$, the $O(F)$ flow past the body illustrated in figure 5 as the basic flow, instead of the $O\left(F^{2}\right)$ flow of Drazin's (1961) theory illustrated in figure 4.

\section{Wave equation}

Having established that, for either weak or strong stratification, lee waves are small-amplitude disturbances to an appropriate base state, and having determined the representation of the sphere in each case, we move on to writing the equations of motion. A fixed frame of reference is used, such that the fluid is at rest at infinity. The system of coordinates $(x, y, z)$ is also fixed, with origin $O$ at the position at $t=0$ of the centre of the sphere. The position $\boldsymbol{x}=(x, y, z)$ in this system, the velocity $\boldsymbol{u}=(u, v, w)$ relative to this frame and, for irrotational flow, the associated velocity potential $\phi$ are related to the position $\boldsymbol{x}_{1}=\left(x_{1}, y, z\right)$, velocity $\boldsymbol{u}_{1}=\left(u_{1}, v, w\right)$ and velocity potential $\phi_{1}$ relative to the sphere by $x_{1}=x+U t, u_{1}=u+U$ and $\phi_{1}=\phi+U x_{1}$, respectively.

In the presence of a source of mass releasing the volume $q$ of fluid per unit volume per unit time, the small-amplitude disturbances $\rho, p$ and $\boldsymbol{u}$ to the density $\rho_{0}$, pressure $p_{0}$ and velocity $\mathbf{0}$ at rest are governed by the linearized equations of motion

$$
\rho_{0} \frac{\partial \boldsymbol{u}}{\partial t}=-\nabla p+\rho \boldsymbol{g}, \quad \nabla \cdot \boldsymbol{u}=q, \quad \frac{\partial \rho}{\partial t}=\rho_{0} \frac{N^{2}}{g} w,
$$

respectively the Euler equation, the equation of continuity and the equation of state, with $\boldsymbol{g}=-g \boldsymbol{e}_{z}$ the acceleration due to gravity and $\boldsymbol{e}_{z}$ a unit vector along the $z$-axis. The wave and vortex components of the motion are separated as discussed by Voisin (2003). The vortex component, containing all the vertical vorticity, is constant and hence zero for motion started from rest. The wave component is expressed as

$$
\rho=\rho_{0} \frac{N^{2}}{g} \frac{\partial^{2}}{\partial t \partial z} \chi, \quad p=-\rho_{0}\left(\frac{\partial^{2}}{\partial t^{2}}+N^{2}\right) \frac{\partial}{\partial t} \chi, \quad \boldsymbol{u}=\left(\frac{\partial^{2}}{\partial t^{2}} \nabla+N^{2} \nabla_{\mathrm{h}}\right) \chi,
$$

in terms of a wave function $\chi$ satisfying the equation of propagation

$$
\left(\frac{\partial^{2}}{\partial t^{2}} \nabla^{2}+N^{2} \nabla_{\mathrm{h}}^{2}\right) \chi=q
$$

In the following, this equation will be solved in terms of the Green's function $G(\boldsymbol{x}, t)$, corresponding to unit impulsive point forcing $q(\boldsymbol{x}, t)=\delta(\boldsymbol{x}) \delta(t)$, and of its Fourier transform $G(\boldsymbol{k}, \omega)$. Both have been calculated by Voisin $(1991 b, 2003)$. From 
(4.3) we obtain immediately

$$
G(\boldsymbol{k}, \omega)=\frac{1}{(\omega+\mathrm{i} 0)^{2} \kappa^{2}-N^{2} \kappa_{\mathrm{h}}^{2}},
$$

where the notation $\omega+\mathrm{i} 0=\lim _{\epsilon \rightarrow 0}(\omega+\mathrm{i} \epsilon)$, with $\epsilon>0$, means that to the frequency is added a positive imaginary part which is later allowed to tend to 0 . This procedure ensures that the radiation condition, in the form of the requirement of causality, is satisfied. Upon introduction of Hadamard's finite part Pf and application of the Sokhotsky-Plemelj formula, the real and imaginary parts of $G(\boldsymbol{k}, \omega)$ separate as

$$
G(\boldsymbol{k}, \omega)=\operatorname{Pf}\left(\frac{1}{\omega^{2} \kappa^{2}-N^{2} \kappa_{\mathrm{h}}^{2}}\right)-\mathrm{i} \pi \delta\left(\omega^{2} \kappa^{2}-N^{2} \kappa_{\mathrm{h}}^{2}\right) \operatorname{sign} \omega .
$$

Inverse Fourier transformation in space, followed by addition of the contributions of the singular frequencies of $G(\boldsymbol{x}, \omega)$ to the inverse transformation in time, yields, at large times when $N|t| \gg 1$,

$$
G(x, t) \sim-\frac{H(t)}{(2 \pi)^{3 / 2} N r_{\mathrm{h}}}\left[\frac{\cos (N t|z| / r-\pi / 4)}{(N t|z| / r)^{1 / 2}}+\frac{\sin (N t-\pi / 4)}{(N t)^{1 / 2}}\right],
$$

namely a superposition of gravity waves of frequency $N|z| / r$ and buoyancy oscillations of frequency $N$.

\section{Wave drag}

\subsection{General expression}

Calculations of the internal wave drag in the literature, for example by Blumen (1965) and Baines $(1995, \S 6.1 .6)$ for the horizontal flow over an obstacle, or by Warren (1960) for the vertical motion of a body and MacKinnon, Mulley \& Warren (1969) for its inclined motion, rely generally on the obstacle or body being flat or slender. In order to deal with a sphere, we use another approach introduced by Gorodtsov \& Teodorovich (1980) for the horizontal motion of a three-dimensional source, Gorodtsov (1980) for its vertical motion, and Gorodtsov \& Teodorovich (1981) for the motion of a twodimensional source. The approach was refined later by Gorodtsov \& Teodorovich (1982, 1983), then extended by Gorodtsov, Reznik \& Stepanyants (1997) to both drag and lift for both two- and three-dimensional sources. It has been applied recently by Scase \& Dalziel (2004) to the inclined motion of a sphere, represented by the appropriate generalization of the source (3.5).

The approach proceeds from the energy equation

$$
\frac{\partial}{\partial t}\left(\frac{1}{2} \rho_{0} \boldsymbol{u}^{2}+\frac{1}{2} \rho_{0} N^{2} \zeta^{2}\right)+\nabla \cdot(p \boldsymbol{u})=p q,
$$

deduced from (4.1) and leading to the identification of $\rho_{0} \boldsymbol{u}^{2} / 2+\rho_{0} N^{2} \zeta^{2} / 2$ as the wave energy density, $p \boldsymbol{u}$ as the wave energy flux, and $p q$ as the work exerted by the source per unit volume per unit time; here $\zeta$, defined by $w=\partial \zeta / \partial t$, is the vertical displacement. For uniform translation of a source $q(\boldsymbol{x}, t)=q_{0}\left(\boldsymbol{x}-\boldsymbol{u}_{0} t\right)$ at the velocity $\boldsymbol{u}_{0}$, the wave energy density is independent of time, implying that the power $P$ lost by the source to the waves may be calculated either by integration of the wave energy flux over a surface encompassing the source path, or by evaluation of the rate of work produced within the whole fluid domain. The equivalence of the two methods has been verified for vertical translation by Gorodtsov \& Teodorovich (1983). 
Applying the second method and using Parseval's theorem, we have

$$
P=\int p(\boldsymbol{x}, t) q(\boldsymbol{x}, t) \mathrm{d}^{3} x=\frac{1}{(2 \pi)^{3}} \int p(\boldsymbol{k}, t) q(-\boldsymbol{k}, t) \mathrm{d}^{3} k,
$$

which for a source of spectrum $q(\boldsymbol{k}, \omega)=2 \pi q_{0}(\boldsymbol{k}) \delta\left(\omega-\boldsymbol{u}_{0} \cdot \boldsymbol{k}\right)$ is evaluated in terms of the Fourier transform $G(\boldsymbol{k}, \omega)$ of the Green's function as

$$
P=\mathrm{i} \frac{\rho_{0}}{(2 \pi)^{3}} \int\left(\boldsymbol{u}_{0} \cdot \boldsymbol{k}\right)\left[N^{2}-\left(\boldsymbol{u}_{0} \cdot \boldsymbol{k}\right)^{2}\right]\left|q_{0}(\boldsymbol{k})\right|^{2} G\left(\boldsymbol{k}, \boldsymbol{u}_{0} \cdot \boldsymbol{k}\right) \mathrm{d}^{3} k .
$$

This power is independent of time and equal to $\left|\boldsymbol{u}_{0}\right|$ times the wave $\operatorname{drag} D$ on the source. Only the imaginary part, odd with respect to the frequency, of $G(\boldsymbol{k}, \omega)$ contributes to the integration. Using (4.5), we obtain

$$
D=\frac{\rho_{0} N^{3}}{8 \pi^{2}\left|\boldsymbol{u}_{0}\right|} \int \frac{\kappa_{\mathrm{h}} m^{2}}{\kappa^{3}}\left|q_{0}(\boldsymbol{k})\right|^{2} \delta\left[\left(\boldsymbol{u}_{0} \cdot \boldsymbol{k}\right)^{2} \kappa^{2}-N^{2} \kappa_{\mathrm{h}}^{2}\right] \mathrm{d}^{3} k,
$$

an integral over the wavenumber surface defined by the combination of the dispersion relation $\omega^{2} \kappa^{2}=N^{2} \kappa_{\mathrm{h}}^{2}$ and Doppler relation $\omega=\boldsymbol{u}_{0} \cdot \boldsymbol{k}$, which is consistent with Lighthill (1967, 1978, §4.12).

For horizontal translation at the velocity $\boldsymbol{u}_{0}=-U \boldsymbol{e}_{x}$, with $\boldsymbol{e}_{x}$ a unit vector along the $x$-axis, the wave drag reduces to

$$
D=\frac{\rho_{0}}{16 \pi^{2}} \sum_{ \pm} \int_{-N / U}^{N / U} \mathrm{~d} k \int_{-\infty}^{\infty} \mathrm{d} l\left(\frac{N^{2} / U^{2}-k^{2}}{k^{2}+l^{2}}\right)^{1 / 2}\left|q_{0}\left(\boldsymbol{k}_{ \pm}\right)\right|^{2},
$$

with

$$
\boldsymbol{k}_{ \pm}=\left[k, l, \pm\left(1+\frac{l^{2}}{k^{2}}\right)^{1 / 2}\left(\frac{N^{2}}{U^{2}}-k^{2}\right)^{1 / 2}\right] .
$$

More generally, for translation at the velocity $U$ at the angle $\alpha$ to the vertical, upon introduction of the spherical polar coordinates $(\kappa, \vartheta, \varphi)$ according to

$$
k=\kappa \sin \vartheta \cos \varphi, \quad l=\kappa \sin \vartheta \sin \varphi, \quad m=\kappa \cos \vartheta,
$$

the wave drag becomes

$$
D=\frac{\rho_{0} N^{2}}{16 \pi^{2} U^{2}} \int_{0}^{\pi} \mathrm{d} \vartheta \int_{0}^{2 \pi} \mathrm{d} \varphi \frac{\cos ^{2} \vartheta}{|\sin \alpha \cos \varphi+\cos \alpha \cot \vartheta|}\left|q_{0}\left(\boldsymbol{k}_{0}\right)\right|^{2},
$$

with

$$
\boldsymbol{k}_{0}=\frac{N}{U} \frac{(\sin \vartheta \cos \varphi, \sin \vartheta \sin \varphi, \cos \vartheta)}{|\sin \alpha \cos \varphi+\cos \alpha \cot \vartheta|}
$$

We apply now each expression to the calculation of the wave drag coefficient $C_{D}=(2 D) /\left(\pi \rho_{0} a^{2} U^{2}\right)$ of the sphere, based on the two representations (3.5) and (3.14) of this sphere, with the notation $q\left(\boldsymbol{x}_{1}\right)$ changed to $q_{0}(\boldsymbol{x})$.

\subsection{Weak stratification}

For weak stratification, corresponding to the source (3.5), a change of variables according to $\xi=U|k| / N=\sin \vartheta$ and $\eta=\kappa_{\mathrm{h}} /|k|=1 /|\cos \varphi|$ yields the single integral

$$
C_{D}=\frac{9 \pi}{8 F} \int_{1}^{\infty} \frac{\mathrm{d} \eta}{\eta^{3}\left(\eta^{2}-1\right)^{1 / 2}} J_{3 / 2}^{2}\left(\frac{\eta}{F}\right) \text {. }
$$



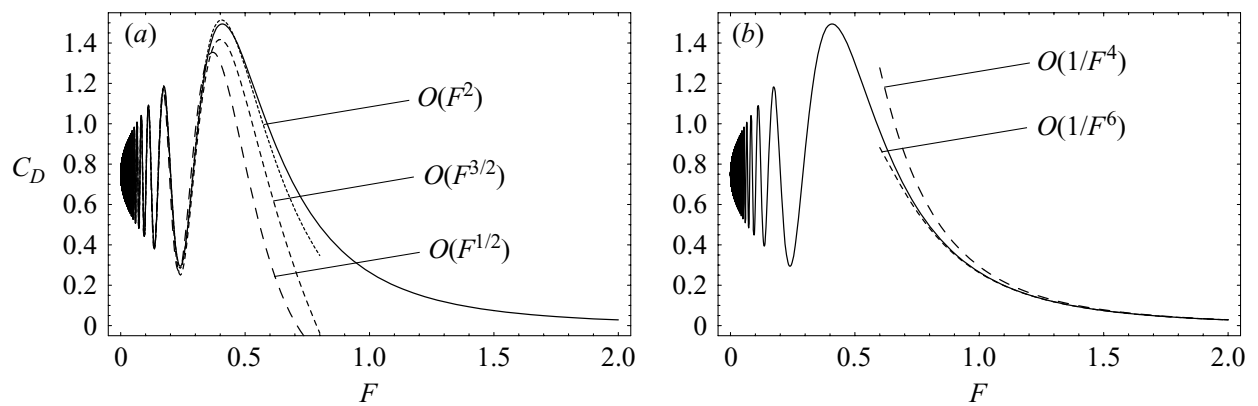

FiguRE 6. Wave drag coefficient $C_{D}$ versus internal Froude number $F$, for weakly stratified flow past a sphere. The exact value of the drag (solid curve) is plotted together with its expansions for either $(a)$ small $F$ or $(b)$ large $F$, to various orders (dashed curves, each accompanied by the indication of the highest order retained).

The variations with $F$ of this integral, first derived by Gorodtsov \& Teodorovich (1982), are plotted in figure 6.

Being based on a representation of the sphere appropriate at $F \gg 1$, this result is only interesting through its expansion as $F \rightarrow \infty$. The leading-order term (1.5a) of the expansion has been given by Gorodtsov \& Teodorovich (1982) with no explanation. Its derivation by standard methods is complicated by the need to separate, as $F \rightarrow \infty$, the global contribution of the majority $\eta=O(1)$ of the range of integration, inside which the Bessel function $J_{3 / 2}(\eta / F)$ may be replaced by its expansion for small $\eta / F$, from the local contribution of the small region $\eta=O(F)$ at infinity, inside which $\left(\eta^{2}-1\right)^{1 / 2}$ may be replaced by its expansion for large $\eta$. As described by Hinch (1991, $\S 3.4$ ), the separation may be achieved by splitting the range of integration into two at an intermediate point $\delta=O\left(F^{1 / 2}\right)$, such that $1 \ll \delta \ll F$. The global contribution is evaluated as

$$
\frac{9 \pi}{8 F} \int_{1}^{\delta} \frac{J_{3 / 2}^{2}(\eta / F)}{\eta^{3}\left(\eta^{2}-1\right)^{1 / 2}} \mathrm{~d} \eta=\frac{1}{4 F^{4}}\left[\ln (2 \delta)-\frac{\delta^{2}}{10 F^{2}}-\frac{1}{4 \delta^{2}}+O\left(\frac{1}{F^{2}}\right)\right],
$$

and the local contribution as

$$
\frac{9 \pi}{8 F} \int_{\delta}^{\infty} \frac{J_{3 / 2}^{2}(\eta / F)}{\eta^{3}\left(\eta^{2}-1\right)^{1 / 2}} \mathrm{~d} \eta=\frac{1}{4 F^{4}}\left[\ln \left(\frac{F}{2 \delta}\right)+\frac{7}{4}-\gamma+\frac{\delta^{2}}{10 F^{2}}+\frac{1}{4 \delta^{2}}+O\left(\frac{1}{F^{2}}\right)\right],
$$

the combination of the two being as expected independent of the artificially introduced $\delta$ and given by $(1.5 a)$. However, the procedure is rather cumbersome, even only for the derivation of this leading-order term, and it would become rapidly intractable should higher-order terms be looked for or should a double integral be considered as later in $\S 5.3$. For this reason we shall use the alternative and more powerful method known as Mellin-Barnes integration.

Mellin-Barnes integrals were introduced in the early twentieth century separately by Mellin (1910), in connection with the theory of integral transforms, and Barnes (1906, 1907, 1908), in connection with the theory of hypergeometric series. Shortly afterwards, they were applied to a number of special functions, including Bessel functions (Watson 1944, $\S \S 6.5-51,7.5-51,13.5$ and 13.6-61), but for the most part they have remained little used in applied mathematics until being revived recently by Bleistein \& Handelsman (1986) and Paris \& Kaminski (2001), among others. In the following we shall adopt the presentation of Paris \& Kaminski, which incorporates the latest extensions of the theory. 
The method is best explained by example. We start from the representation of the square of the Bessel function $J_{v}(x)$ as the Mellin-Barnes integral

$$
J_{v}^{2}(x)=\frac{1}{2 \mathrm{i} \pi^{3 / 2}} \int_{-\mathrm{i} \infty}^{+\mathrm{i} \infty} \frac{\Gamma(s) \Gamma(1 / 2+v-s)}{\Gamma(1+v-s) \Gamma(1+2 v-s)} x^{2 v-2 s} \mathrm{~d} s,
$$

deduced from the combination of Watson $(1944, \S 13.61)$ with the duplication formula. This representation is valid for $x>0$ and $\operatorname{Re} v \geqslant-3 / 4$, the integral being absolutely convergent for $\operatorname{Re} v>-1 / 4$ and semi-convergent for $\operatorname{Re} v \geqslant-3 / 4$. The contour of integration is the imaginary axis, indented to leave the simple poles $s=-n$ of $\Gamma(s)$ on the left, with $n=0,1,2, \ldots$, and $s=v+1 / 2+n$ of $\Gamma(1 / 2+v-s)$ on the right. Substitution in (5.10) followed by interchange of the order of integration yields

$$
C_{D}=\frac{9 \pi}{16 F^{4}} \frac{1}{2 \mathrm{i} \pi} \int_{-\mathrm{i} \infty}^{+\mathrm{i} \infty} \frac{\Gamma^{2}(s) \Gamma(2-s)}{\Gamma(s+1 / 2) \Gamma(5 / 2-s) \Gamma(4-s)} F^{2 s} \mathrm{~d} s,
$$

or alternatively, by the reflection formula,

$$
C_{D}=\frac{9 \pi}{16 F^{4}} \frac{1}{2 \mathrm{i} \pi} \int_{-\mathrm{i} \infty}^{+\mathrm{i} \infty} \frac{\Gamma^{2}(s)}{(s-1 / 2)(s-3 / 2)(s-2)(s-3)} \cos (s \pi) F^{2 s} \mathrm{~d} s .
$$

In both expressions, the integrand has an infinite sequence of double poles $s=-n$ on the left of the contour, and two simple poles $s=2,3$ on the right.

The asymptotic formula $\Gamma(a s+b) \sim(2 \pi)^{1 / 2} \mathrm{e}^{-a s}(a s)^{a s+b-1 / 2}$, valid for arbitrary constants $a$ and $b$ as $|s| \rightarrow \infty$ with $|\arg s|<\pi$ and $a>0$, implies that, at infinity along any contour parallel to the imaginary axis, namely as $|\tau| \rightarrow \infty$ with $s=\sigma+\mathrm{i} \tau$, we have

$$
\Gamma(a s+b) \sim(2 \pi)^{1 / 2} \mathrm{e}^{-\pi a|\tau| / 2}(a|\tau| / \mathrm{e})^{\mathrm{i} a \tau}(\mathrm{i} a \tau)^{a \sigma+b-1 / 2} .
$$

In the same limit, we also have $\cos (a s \pi) \sim \mathrm{e}^{\pi a|\tau|} / 2$. Accordingly, the integrand of (5.15) has the algebraic behaviour $|\tau|^{2 \sigma-5}(F|\tau| / \mathrm{e})^{2 \mathrm{i} \tau}$ as $|\tau| \rightarrow \infty$, implying that the contour of integration may be displaced at infinity to the left, but not beyond the 'barrier' $\operatorname{Re} s=5 / 2$ to the right.

Displacement to the left over the poles on the negative real axis yields the series expansion

$$
\begin{aligned}
C_{D}= & \frac{9}{2 F^{4}} \sum_{n=0}^{\infty} \frac{(-1)^{n}}{F^{2 n}} \frac{1}{(n !)^{2}(2 n+1)(2 n+3)(n+2)(n+3)} \\
& \times\left[\ln F+\psi(n+1)+\frac{1}{2 n+1}+\frac{1}{2 n+3}+\frac{1}{2 n+4}+\frac{1}{2 n+6}\right],
\end{aligned}
$$

of which $(1.5 a)$ is the leading order and which becomes, to next order,

$$
C_{D}=\frac{\ln F+7 / 4-\gamma}{4 F^{4}}-\frac{\ln F+73 / 40-\gamma}{40 F^{6}}+O\left(\frac{1}{F^{8}}\right),
$$

with $\psi(x)$ the digamma function. This expansion is compared with the numerical determination of $C_{D}$ in figure $6(b)$.

The corresponding expansion as $F \rightarrow 0$, though physically meaningless, is also of interest, first to emphasize the ability of the method to account for the oscillations visible in figure 6, and secondly to delineate how the method is applied in this case, in a simpler example than will occur in $\S 5.3$ for strong stratification, when the limit $F \rightarrow 0$ will become physically meaningful. 
The existence of a 'contour barrier' is indicative of the oscillatory behaviour of a Mellin-Barnes integral. To deal with it, we apply the procedure described in $\S 5.6$ of Paris \& Kaminski (2001). In (5.15), we displace the contour of integration to the right, over the pole $s=2$ to the new position $\operatorname{Re} s=c$, with $2<c<5 / 2$. Then we write $\cos (s \pi)=2 \cos ^{2}(s \pi / 2)-1$, so as to separate $C_{D}$ into two parts according to

$$
C_{D}=C_{1}+C_{2} \text {. }
$$

In the first part

$$
C_{1}=\frac{3}{4}-\frac{9 \pi}{16 F^{4}} \frac{1}{2 \mathrm{i} \pi} \int_{c-\mathrm{i} \infty}^{c+\mathrm{i} \infty} \frac{\Gamma^{2}(s)}{(s-1 / 2)(s-3 / 2)(s-2)(s-3)} F^{2 s} \mathrm{~d} s,
$$

the integrand has the same pole $s=3$ to the right of the contour as for $C_{D}$, but exponentially small behaviour $\mathrm{e}^{-\pi|\tau|}|\tau|^{2 \sigma-5}(F|\tau| / \mathrm{e})^{2 \mathrm{i} \tau}$ as $|\tau| \rightarrow \infty$. In the second part

$$
C_{2}=\frac{9 \pi}{8 F^{4}} \frac{1}{2 \mathrm{i} \pi} \int_{c-\mathrm{i} \infty}^{c+\mathrm{i} \infty} \frac{\Gamma^{2}(s)}{(s-1 / 2)(s-3 / 2)(s-2)(s-3)} \cos ^{2}\left(s \frac{\pi}{2}\right) F^{2 s} \mathrm{~d} s,
$$

the integrand has the same algebraic behaviour as $|\tau| \rightarrow \infty$ as for $C_{D}$, but no singularity to the right of the contour.

For $C_{1}$, we simply displace the contour to the right over the pole $s=3$ to obtain

$$
C_{1}=\frac{3}{4}+\frac{3}{5} F^{2}
$$

For $C_{2}$, we deform the contour to a loop $L$, having endpoints at infinity in the half-plane $\operatorname{Re} s<0$, starting in the quarter-plane $\operatorname{Im} s<0$, intersecting the positive real axis at large $\operatorname{Re} s>0$ and finishing in the quarter-plane $\operatorname{Im} s>0$, so that $|s|$ is everywhere large on $L$. Hence, substitution of the inverse factorial expansion

$$
\frac{\Gamma^{2}(s) \Gamma(s-3 / 2) \Gamma(s-3)}{\Gamma(s+1 / 2) \Gamma(s-1)} \sim \pi^{1 / 2} 2^{11 / 2-2 s} \sum_{n=0}^{\infty}(-1)^{n} c_{n} \Gamma\left(2 s-\frac{9}{2}-n\right),
$$

obtained by the method described in $\S 2.2$ of Paris \& Kaminski (2001), is allowed. Its coefficients satisfy the recurrence relation

$$
c_{0}=1, \quad c_{n}=-\frac{1}{4 n} \sum_{m=0}^{n-1} e_{n m} c_{m} \quad(n \geqslant 1)
$$

with

$$
\begin{aligned}
e_{n m}=\frac{2}{9} \frac{\Gamma(n+11 / 2)}{\Gamma(m+7 / 2)}[1-\psi(n+ & \left.\left.\frac{11}{2}\right)+\psi\left(m+\frac{7}{2}\right)\right] \\
& +\frac{8}{27} \frac{\Gamma(n+5 / 2)}{\Gamma(m+1 / 2)}-\frac{14}{27} \frac{\Gamma(n-1 / 2)}{\Gamma(m-5 / 2)},
\end{aligned}
$$

so that, in particular,

$$
c_{0}=1, \quad c_{1}=-\frac{15}{8}, \quad c_{2}=\frac{233}{128}, \quad c_{3}=\frac{6587}{1024} .
$$

Use of the integrals

$$
\begin{gathered}
\frac{1}{2 \mathrm{i} \pi} \int_{L} \Gamma(s-\alpha) z^{-s} \mathrm{~d} s=z^{-\alpha} \exp (-z) \\
\frac{1}{2 \mathrm{i} \pi} \int_{L} \Gamma(s-\alpha)\left(\begin{array}{c}
\cos \\
\sin
\end{array}\right)\left(s \frac{\pi}{2}\right) z^{-s} \mathrm{~d} s=z^{-\alpha}\left(\begin{array}{c}
\cos \\
\sin
\end{array}\right)\left(z+\alpha \frac{\pi}{2}\right),
\end{gathered}
$$



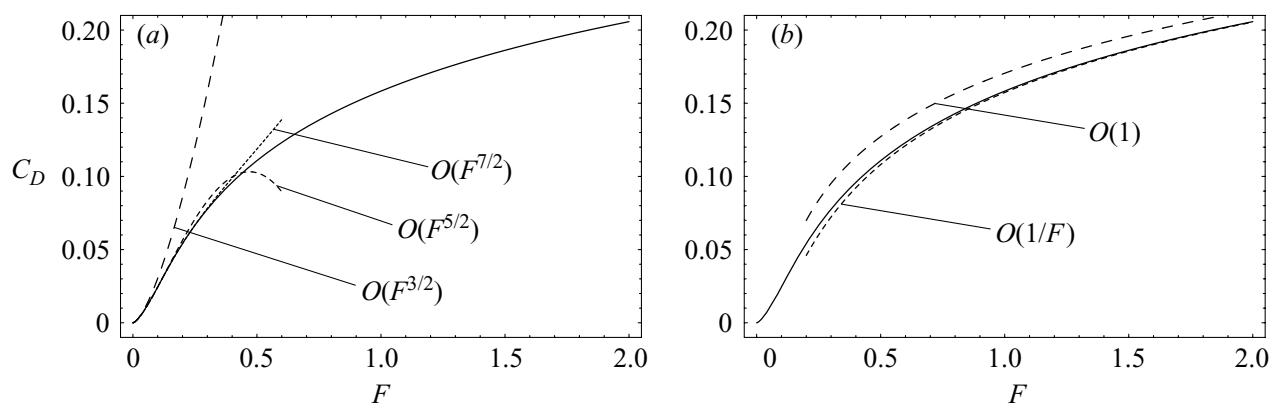

Figure 7. Wave drag coefficient $C_{D}$ versus internal Froude number $F$, for strongly stratified flow past a sphere. The mode of representation is the same as in figure 6 .

taken from $\S 3.3$ of Paris \& Kaminski (2001) and valid for all $z$ and arbitrary $\alpha$ provided the loop $L$ embraces all the poles of $\Gamma(s-\alpha)$, yields immediately

$$
C_{2} \sim \frac{9}{16}(\pi F)^{1 / 2} \sum_{n=0}^{\infty}(-1)^{n} c_{n}\left(\frac{F}{2}\right)^{n}\left[\exp \left(-\frac{2}{F}\right)+\cos \left(\frac{2}{F}+\frac{\pi}{4}+n \frac{\pi}{2}\right)\right] .
$$

Adding the two parts and omitting the exponentially small terms in $C_{2}$, we obtain the asymptotic expansion

$$
C_{D} \sim \frac{3}{4}+\frac{3}{5} F^{2}+\frac{9}{16}(\pi F)^{1 / 2} \sum_{n=0}^{\infty}(-1)^{n} c_{n}\left(\frac{F}{2}\right)^{n} \cos \left(\frac{2}{F}+\frac{\pi}{4}+n \frac{\pi}{2}\right)
$$

with first few orders

$$
C_{D}=\frac{3}{4}+\frac{9}{16}(\pi F)^{1 / 2} \cos \left(\frac{2}{F}+\frac{\pi}{4}\right)-\frac{135}{256} \pi^{1 / 2} F^{3 / 2} \sin \left(\frac{2}{F}+\frac{\pi}{4}\right)+\frac{3}{5} F^{2}+O\left(F^{5 / 2}\right),
$$

which are compared with the numerical determination of $C_{D}$ in figure $6(a)$.

\subsection{Strong stratification}

For strong stratification, owing to the presence of the middle absorbing layer, the two contributions of the top and bottom portions of the sphere, represented by the sources $q_{+}$and $q_{-}$from (3.14), respectively, may be superposed incoherently. The wave drag coefficient is given by the double integral

$$
C_{D}=\frac{32}{\pi} F^{2} \int_{0}^{1} \frac{\mathrm{d} \xi}{\xi^{2}} \int_{1}^{\infty} \frac{\mathrm{d} \eta}{\eta^{4}}\left(\frac{1-\xi^{2}}{\eta^{2}-1}\right)^{1 / 2} J_{2}^{2}\left[\xi \eta\left(\frac{2}{F}\right)^{1 / 2}\right],
$$

whose variations with $F$ are plotted in figure 7 .

To derive its expansion as $F \rightarrow 0$, we turn as above to Mellin-Barnes integration. Substitution of (5.13) followed by interchange of the order of integration yields

$$
C_{D}=\frac{16}{\pi^{1 / 2}} \frac{1}{2 \mathrm{i} \pi} \int_{-\mathrm{i} \infty}^{+\mathrm{i} \infty} \frac{\Gamma^{2}(s) \Gamma(3 / 2-s) \Gamma(5 / 2-s)}{\Gamma(s+1 / 2) \Gamma^{2}(3-s) \Gamma(5-s)}\left(\frac{F}{2}\right)^{s} \mathrm{~d} s,
$$

or alternatively, by the reflection formula,

$$
C_{D}=-\frac{16}{\pi^{3 / 2}} \frac{1}{2 \mathrm{i} \pi} \int_{-\mathrm{i} \infty}^{+\mathrm{i} \infty} \frac{\Gamma^{2}(s) \Gamma^{2}(s-2) \Gamma(s-4)}{\Gamma(s+1 / 2) \Gamma(s-1 / 2) \Gamma(s-3 / 2)} \frac{\sin ^{3}(s \pi)}{\cos ^{2}(s \pi)}\left(\frac{F}{2}\right)^{s} \mathrm{~d} s .
$$

The integrand has an infinite sequence of double poles $s=-n$ on the left of the contour, with $n=0,1,2, \ldots$, and a simple pole $s=3 / 2$ and an infinite sequence 
of double poles $s=5 / 2+n$ on the right. At infinity along any path parallel to the imaginary axis, that is as $|\tau| \rightarrow \infty$ with $s=\sigma+\mathrm{i} \tau$, the integrand has the algebraic behaviour $|\tau|^{2 \sigma-15 / 2}(|\tau| / \mathrm{e})^{2 \mathrm{i} \tau}(F / 2)^{\mathrm{i} \tau}$. Accordingly, the contour of integration may be displaced at infinity to the left, but no further than the barrier $\operatorname{Re} s=15 / 4$ to the right.

Displacement to the left over the poles on the negative real axis yields the series expansion

$$
\begin{aligned}
C_{D} & =\frac{16}{\pi^{3 / 2}} \sum_{n=0}^{\infty}(-1)^{n}\left(\frac{2}{F}\right)^{n} \frac{\Gamma(n+1 / 2) \Gamma(n+3 / 2) \Gamma(n+5 / 2)}{(n !)^{2}[(n+2) !]^{2}(n+4) !}\left[\ln \left(\frac{F}{2}\right)+2 \psi(n+1)\right. \\
& \left.+2 \psi(n+3)+\psi(n+5)-\psi\left(n+\frac{1}{2}\right)-\psi\left(n+\frac{3}{2}\right)-\psi\left(n+\frac{5}{2}\right)\right], \quad(5.35)
\end{aligned}
$$

which is more effective, for the evaluation of $C_{D}$ in Mathematica, than the double integral (5.32) as soon as, say, $F>0.02$. To the first two orders,

$$
C_{D}=\frac{\ln F+5 / 12-2 \gamma+5 \ln 2}{16}-\frac{\ln F+13 / 60-2 \gamma+5 \ln 2}{192 F}+O\left(\frac{1}{F^{2}}\right),
$$

which is compared in figure 7(b) with the numerical evaluation of $C_{D}$.

The expansion of $C_{D}$ at small $F$ follows from the same procedure as in $\S 5.2$. In (5.34), the contour of integration is displaced to the right, over the pole $s=3 / 2$ to the new position $\operatorname{Re} s=c$, with $2<c<5 / 2$. Then the identity $\sin ^{2}(s \pi)=1-\cos ^{2}(s \pi)$ is used to separate $C_{D}$ into two parts according to

$$
C_{D}=C_{1}+C_{2} .
$$

In the first part

$$
C_{1}=\frac{2^{11 / 2}}{15 \pi} F^{3 / 2}-\frac{16}{\pi^{3 / 2}} \frac{1}{2 \mathrm{i} \pi} \int_{c-\mathrm{i} \infty}^{c+\mathrm{i} \infty} \frac{\Gamma^{2}(s) \Gamma^{2}(s-2) \Gamma(s-4)}{\Gamma(s+1 / 2) \Gamma(s-1 / 2) \Gamma(s-3 / 2)} \frac{\sin (s \pi)}{\cos ^{2}(s \pi)}\left(\frac{F}{2}\right)^{s} \mathrm{~d} s,
$$

the integrand has the same singularities to the right of the contour as for $C_{D}$, but exponentially small behaviour $\mathrm{e}^{-2 \pi|\tau|}|\tau|^{2 \sigma-15 / 2}(|\tau| / \mathrm{e})^{2 i \tau}(F / 2)^{\mathrm{i} \tau}$ as $|\tau| \rightarrow \infty$. Displacement of the contour to the right over the poles on the positive real axis yields

$$
\begin{aligned}
C_{1} \sim & \frac{2^{11 / 2}}{15 \pi} F^{3 / 2}-\frac{2^{3 / 2}}{\pi^{7 / 2}} F^{5 / 2} \sum_{n=0}^{\infty}(-1)^{n}\left(\frac{F}{2}\right)^{n} \frac{\Gamma(n-3 / 2) \Gamma^{2}(n+1 / 2) \Gamma^{2}(n+5 / 2)}{n !(n+1) !(n+2) !} \\
\times & {\left[\ln \left(\frac{2}{F}\right)+\psi(n+1)+\psi(n+2)+\psi(n+3)\right.} \\
& \left.\quad-\psi\left(n-\frac{3}{2}\right)-2 \psi\left(n+\frac{1}{2}\right)-2 \psi\left(n+\frac{5}{2}\right)\right] .
\end{aligned}
$$

In the second part

$$
C_{2}=\frac{16}{\pi^{3 / 2}} \frac{1}{2 \mathrm{i} \pi} \int_{c-\mathrm{i} \infty}^{c+\mathrm{i} \infty} \frac{\Gamma^{2}(s) \Gamma^{2}(s-2) \Gamma(s-4)}{\Gamma(s+1 / 2) \Gamma(s-1 / 2) \Gamma(s-3 / 2)} \sin (s \pi)\left(\frac{F}{2}\right)^{s} \mathrm{~d} s,
$$

the integrand has the same algebraic behaviour as $|\tau| \rightarrow \infty$ as for $C_{D}$, but no singularity to the right of the contour. Substitution of the inverse factorial expansion

$$
\frac{\Gamma^{2}(s) \Gamma^{2}(s-2) \Gamma(s-4)}{\Gamma(s+1 / 2) \Gamma(s-1 / 2) \Gamma(s-3 / 2)} \sim \pi^{1 / 2} 2^{8-2 s} \sum_{n=0}^{\infty}(-1)^{n} c_{n} \Gamma(2 s-7-n),
$$


whose coefficients satisfy the recurrence relation (5.24) with

$$
\begin{aligned}
e_{n m}= & \frac{3}{64} \frac{(n+7) !}{(m+5) !}\left[\frac{7}{24}-\psi(n+8)+\psi(m+6)\right] \\
& +\frac{15}{32} \frac{(n+3) !}{(m+1) !}\left[\frac{77}{60}-\psi(n+4)+\psi(m+2)\right]-\frac{315}{512} \frac{(n-1) !}{(m-3) !}
\end{aligned}
$$

so that, in particular,

$$
c_{0}=1, \quad c_{1}=0, \quad c_{2}=\frac{9}{2}, \quad c_{3}=33,
$$

followed by use of the integral (5.28), yields

$$
C_{2} \sim-\frac{2^{1 / 2}}{\pi} F^{7 / 2} \sum_{n=0}^{\infty}(-1)^{n} c_{n}\left(\frac{F}{8}\right)^{n / 2} \cos \left(\frac{2^{3 / 2}}{F^{1 / 2}}+n \frac{\pi}{2}\right) .
$$

Adding the two parts and retaining the first few orders, we obtain the asymptotic expansion

$$
\begin{aligned}
C_{D}= & \frac{2^{11 / 2}}{15 \pi} F^{3 / 2}-\frac{3}{2^{3 / 2} \pi} F^{5 / 2}\left[\ln \left(\frac{1}{F}\right)-\frac{11}{2}+2 \gamma+11 \ln 2\right]-\frac{75}{2^{17 / 2} \pi} F^{7 / 2} \\
& \times\left[\ln \left(\frac{1}{F}\right)-\frac{39}{5}+2 \gamma+11 \ln 2\right]-\frac{2^{1 / 2}}{\pi} F^{7 / 2} \cos \left(\frac{2^{3 / 2}}{F^{1 / 2}}\right)+O\left(F^{9 / 2}\right),
\end{aligned}
$$

compared in figure $7(a)$ with the numerical evaluation of $C_{D}$.

To leading order, the small- $F$ expansion becomes

$$
C_{D} \sim \frac{32 \sqrt{ } 2}{15 \pi} F^{3 / 2} \approx 0.96 F^{3 / 2}
$$

Not only does it confirm the variation as $F^{3 / 2}$ derived by Greenslade (2000) in (1.6a), but also it predicts the value of the constant $B$ in front of the variation, 0.96 , within the error bounds of the values deduced in table 1 from the experiments of Mason (1977), Lofquist \& Purtell (1984), Shishkina (1996) and Vosper et al. (1999).

The consistency of the two expansions of the wave drag coefficient, (5.18) for $F \gg 1$ and (5.45) for $F \ll 1$, with the analysis by Greenslade (2000) of the available experimental measurements of the drag validates the underlying representations (3.5) and (3.14) of the sphere, and legitimates their use for the calculation of the waves themselves.

\section{Wave field}

\subsection{General expression}

The calculation of the waves from a point monopole $q(\boldsymbol{x}, t)=H(t) Q(t) \delta\left[\boldsymbol{x}-\boldsymbol{x}_{0}(t)\right]$, starting at time $t=0$ moving along the path $x_{0}(t)$ with non-oscillatory strength $Q(t)$, has been performed by Voisin $(1991 a, 1994)$. Its extension to a source of finite size $a$, of the form $q(\boldsymbol{x}, t)=H(t) q_{0}\left[\boldsymbol{x}-\boldsymbol{x}_{0}(t), t\right]$ with $q_{0}(\boldsymbol{x}, t)$ vanishing rapidly for $r \gg a$ and not oscillating with $t$, has been sketched by Voisin (1991a) and used by Dupont \& Voisin (1996), Scase \& Dalziel (2004) and Broutman \& Rottman (2004), but so far has remained unpublished. We present the extension briefly, for application to the sphere. 
The solution of the wave equation (4.3) is written in terms of the Green's function $G(\boldsymbol{x}, t)$ as the convolution integral

$$
\chi(\boldsymbol{x}, t)=\int_{0}^{t} \mathrm{~d} t^{\prime} \int \mathrm{d}^{3} x^{\prime} q_{0}\left(\boldsymbol{x}^{\prime}, t^{\prime}\right) G\left[\boldsymbol{x}-\boldsymbol{x}_{0}\left(t^{\prime}\right)-\boldsymbol{x}^{\prime}, t-t^{\prime}\right],
$$

namely as a superposition of impulses emitted at the various elapsed times $0<t^{\prime}<t$ by the various points $\boldsymbol{x}_{0}\left(t^{\prime}\right)+\boldsymbol{x}^{\prime}$ of the source at the various positions $\boldsymbol{x}_{0}\left(t^{\prime}\right)$ of the reference point of this source along its path; hereinafter, a factor $H(t)$ is implicit in the expression for the waves.

At large propagation times compared with the buoyancy period, for $N\left(t-t^{\prime}\right) \gg 1$, we may replace the Green's function by its asymptotic expansion (4.6) to obtain

$$
\chi(\boldsymbol{x}, t) \sim-\frac{1}{(2 \pi)^{3 / 2} N} \int_{0}^{t} \mathrm{~d} t^{\prime} \int \mathrm{d}^{3} x^{\prime} \frac{q_{0}\left(\boldsymbol{x}^{\prime}, t^{\prime}\right)}{\left|\boldsymbol{X}_{\mathrm{h}}\left(t^{\prime}\right)-\boldsymbol{x}_{\mathrm{h}}^{\prime}\right|} \frac{\cos \left[N\left(t-t^{\prime}\right) \frac{\left|Z\left(t^{\prime}\right)-z^{\prime}\right|}{\left|\boldsymbol{X}\left(t^{\prime}\right)-\boldsymbol{x}^{\prime}\right|}-\frac{\pi}{4}\right]}{\left[N\left(t-t^{\prime}\right) \frac{\left|Z\left(t^{\prime}\right)-z^{\prime}\right|}{\left|\boldsymbol{X}\left(t^{\prime}\right)-\boldsymbol{x}^{\prime}\right|}\right]^{1 / 2}},
$$

with $\boldsymbol{X}\left(t^{\prime}\right)=\boldsymbol{x}-\boldsymbol{x}_{0}\left(t^{\prime}\right)$ the position at $t^{\prime}$ of the observation point relative to the source. Buoyancy oscillations, which are subdominant compared with gravity waves in the physical disturbances, such as density, pressure and velocity, associated with the Green's function (Voisin 1991b), have been omitted.

In the gravity waves (6.2), the phase of the integrand varies with $\boldsymbol{x}^{\prime}$ much more rapidly than the amplitude. Hence, at large distances from the source compared with its size, for $R\left(t^{\prime}\right) \gg a$, we may expand the phase as

$$
-N\left(t-t^{\prime}\right) \frac{\left|Z\left(t^{\prime}\right)-z^{\prime}\right|}{\left|\boldsymbol{X}\left(t^{\prime}\right)-\boldsymbol{x}^{\prime}\right|} \sim-N\left(t-t^{\prime}\right) \frac{\left|Z\left(t^{\prime}\right)\right|}{R\left(t^{\prime}\right)}-\boldsymbol{k}\left(t^{\prime}\right) \cdot \boldsymbol{x}^{\prime},
$$

in terms of the wavenumber vector

$$
\boldsymbol{k}\left(t^{\prime}\right)=\nabla\left[-N\left(t-t^{\prime}\right) \frac{\left|Z\left(t^{\prime}\right)\right|}{R\left(t^{\prime}\right)}\right]=N\left(t-t^{\prime}\right) \frac{\left|Z\left(t^{\prime}\right)\right|}{R^{2}\left(t^{\prime}\right)}\left[\frac{X\left(t^{\prime}\right)}{R\left(t^{\prime}\right)}-\frac{R\left(t^{\prime}\right)}{Z\left(t^{\prime}\right)} \boldsymbol{e}_{z}\right],
$$

while setting $\boldsymbol{x}^{\prime}=\mathbf{0}$ in the amplitude. We obtain

$$
\chi(\boldsymbol{x}, t) \sim-\frac{\operatorname{Re}}{(2 \pi)^{3 / 2} N} \int_{0}^{t} \frac{q_{0}\left[\boldsymbol{k}\left(t^{\prime}\right), t^{\prime}\right]}{R_{\mathrm{h}}\left(t^{\prime}\right)} \frac{\exp \left\{-\mathrm{i}\left[N\left(t-t^{\prime}\right) \frac{\left|Z\left(t^{\prime}\right)\right|}{R\left(t^{\prime}\right)}-\frac{\pi}{4}\right]\right\}}{\left[N\left(t-t^{\prime}\right) \frac{\left|Z\left(t^{\prime}\right)\right|}{R\left(t^{\prime}\right)}\right]^{1 / 2}} \mathrm{~d} t^{\prime} .
$$

The spatial integration has resulted in the replacement of the source by its spectrum, and the only remaining integration is with respect to time.

At large times after the start-up compared with the buoyancy period, for $N t \gg 1$, the method of stationary phase may be applied to this integration. The point of stationary phase $t_{\mathrm{s}}$, defined by the implicit equation

$$
\frac{R\left(t_{\mathrm{s}}\right)}{t-t_{\mathrm{s}}}=\boldsymbol{u}_{0}\left(t_{\mathrm{s}}\right) \cdot\left[\frac{\boldsymbol{X}\left(t_{\mathrm{s}}\right)}{R\left(t_{\mathrm{s}}\right)}-\frac{R\left(t_{\mathrm{s}}\right)}{Z\left(t_{\mathrm{s}}\right)} \boldsymbol{e}_{z}\right],
$$

with $\boldsymbol{u}_{0}(t)=\mathrm{d} \boldsymbol{x}_{0} / \mathrm{d} t$ the velocity of the source, represents the time when the source has emitted, at position $\boldsymbol{x}_{0}\left(t_{\mathrm{s}}\right)$, the waves received later at time $t$ at position $\boldsymbol{x}$. The 
wave function becomes

$$
\chi(\boldsymbol{x}, t) \sim \frac{1}{2 \pi N^{2}|A|^{1 / 2}} \frac{R}{R_{\mathrm{h}}|Z|} \operatorname{Im}\left\{q_{0}\left(\boldsymbol{k}, t_{\mathrm{s}}\right) \exp \left\{\mathrm{i}\left[\Phi-\frac{\pi}{2} H(A)\right]\right\}\right\},
$$

where all quantities are evaluated implicitly at the retarded time, including the phase

$$
\Phi=-N\left(t-t_{\mathrm{s}}\right) \frac{|Z|}{R}=-\frac{N}{c_{\mathrm{g}}}|Z|=-\omega\left(t-t_{\mathrm{s}}\right),
$$

the frequency and wavenumber vector

$$
\omega=-\frac{\partial \Phi}{\partial t}=N \frac{|Z|}{R}, \quad \boldsymbol{k}=\nabla \Phi=\frac{\omega}{c_{\mathrm{g}}}\left(\frac{\boldsymbol{X}}{R}-\frac{R}{Z} \boldsymbol{e}_{z}\right),
$$

the group velocity

$$
\boldsymbol{c}_{\mathrm{g}}=\frac{\boldsymbol{X}}{t-t_{\mathrm{s}}}=\boldsymbol{u}_{0} \cdot\left(\frac{X}{R}-\frac{R}{Z} \boldsymbol{e}_{z}\right) \frac{X}{R}
$$

and the factor

$$
A=R \frac{\boldsymbol{\gamma}_{0}}{c_{\mathrm{g}}^{2}} \cdot\left(\frac{\boldsymbol{X}}{R}-\frac{R}{Z} \boldsymbol{e}_{z}\right)-\left(\frac{\boldsymbol{u}_{0}}{c_{\mathrm{g}}} \times \frac{\boldsymbol{X}}{R}\right)^{2}+2 \frac{\boldsymbol{u}_{0}}{c_{\mathrm{g}}} \cdot \frac{R}{Z} \boldsymbol{e}_{z},
$$

with $\gamma_{0}(t)=\mathrm{d}^{2} x_{0} / \mathrm{d} t^{2}$ the acceleration of the source. The assumption of large propagation times $N\left(t-t_{\mathrm{s}}\right) \gg 1$ is seen to amount to that of large distances from the source compared with the wavelength $\kappa R \gg 1$. Hence, in total, the asymptotic expression for the waves requires $N t \gg 1, R \gg a$ and $\kappa R \gg 1$.

The density, pressure and velocity follow from the differentiation of the wave function according to (4.2), taking into account that, to leading order, only the variations of the phase, governed by (6.9), are significant. The vertical displacement is obtained as

$$
\zeta(x, t) \sim-\frac{\operatorname{sign} Z}{2 \pi c_{\mathrm{g}}|A|^{1 / 2}} \frac{R_{\mathrm{h}}}{R^{2}} \operatorname{Im}\left\{q_{0}\left(\boldsymbol{k}, t_{\mathrm{s}}\right) \exp \left\{\mathrm{i}\left[\Phi-\frac{\pi}{2} H(A)\right]\right\}\right\} .
$$

We apply this result to the representations (3.1), (3.5) and (3.14) of the sphere, with the notation $q\left(\boldsymbol{x}_{1}\right)$ changed to $q_{0}(\boldsymbol{x})$.

\subsection{Horizontal motion}

For uniform horizontal translation $\boldsymbol{x}_{0}=-U t \boldsymbol{e}_{x}$ at the velocity $\boldsymbol{u}_{0}=-U \boldsymbol{e}_{x}$, upon introduction of spherical polar coordinates $\left(r_{1}, \vartheta_{1}, \varphi_{1}\right)$ relative to the current position of the source and of axis its path, namely

$$
x_{1}=x+U t=r_{1} \cos \vartheta_{1}, \quad y=r_{1} \sin \vartheta_{1} \cos \varphi_{1}, \quad z=r_{1} \sin \vartheta_{1} \sin \varphi_{1},
$$

the solution of the retarded time equation (6.6) is

$$
t-t_{\mathrm{s}}=\frac{r_{1}}{U \cos \vartheta_{1}}
$$

implying, by the condition of causality $t_{\mathrm{s}}<t$, that the waves are only found downstream, in the half-space $x_{1}>0$. The path travelled by the waves between emission and reception is

$$
\boldsymbol{X}=r_{1} \tan \vartheta_{1}\left(-\sin \vartheta_{1}, \cos \vartheta_{1} \cos \varphi_{1}, \cos \vartheta_{1} \sin \varphi_{1}\right),
$$

in terms of which we obtain immediately the frequency

$$
\omega=N \cos \vartheta_{1}\left|\sin \varphi_{1}\right|
$$


the wavenumber vector

$$
\boldsymbol{k}=\frac{N}{U}\left(-\cos \vartheta_{1}\left|\sin \varphi_{1}\right|, \frac{\cos ^{2} \vartheta_{1}}{\sin \vartheta_{1}} \cos \varphi_{1}\left|\sin \varphi_{1}\right|,-\frac{\sin ^{2} \vartheta_{1}+\cos ^{2} \vartheta_{1} \cos ^{2} \varphi_{1}}{\sin \vartheta_{1}} \operatorname{sign} z\right)
$$

the group velocity

$$
\boldsymbol{c}_{\mathrm{g}}=U \sin \vartheta_{1}\left(-\sin \vartheta_{1}, \cos \vartheta_{1} \cos \varphi_{1}, \cos \vartheta_{1} \sin \varphi_{1}\right),
$$

and the vertical displacement

$$
\zeta(\boldsymbol{x}, t) \sim-\frac{\operatorname{sign} z}{2 \pi U r_{1}}\left(1+\cot ^{2} \vartheta_{1} \cos ^{2} \varphi_{1}\right)^{1 / 2} \operatorname{Im}\left[q_{0}\left(\boldsymbol{k}, t_{\mathrm{s}}\right) \exp \left(-\mathrm{i} \frac{N}{U} r_{1}\left|\sin \varphi_{1}\right|\right)\right],
$$

in which a factor $H\left(x_{1}\right)$ is implicit.

Considering the three possible representations of the sphere, we have, in particular, for the dipole (3.1),

$$
\zeta(\boldsymbol{x}, t) \sim \frac{N a^{3}}{U r_{1}} \cos \vartheta_{1} \sin \varphi_{1}\left(1+\cot ^{2} \vartheta_{1} \cos ^{2} \varphi_{1}\right)^{1 / 2} \cos \left(\frac{N}{U} r_{1} \sin \varphi_{1}\right)
$$

for the weakly stratified model (3.5),

$$
\zeta(\boldsymbol{x}, t) \sim 3 \frac{a^{2}}{r_{1}} \cos \vartheta_{1} \sin \varphi_{1} j_{1}\left[\frac{N a}{U}\left(1+\cot ^{2} \vartheta_{1} \cos ^{2} \varphi_{1}\right)^{1 / 2}\right] \cos \left(\frac{N}{U} r_{1} \sin \varphi_{1}\right)
$$

and for the strongly stratified model (3.14),

$$
\begin{aligned}
\zeta(\boldsymbol{x}, t) \sim 2 \frac{U^{2}}{N^{2} r_{1}} \frac{J_{2}\left[(2 N a / U)^{1 / 2} \cos \vartheta_{1} \sin \varphi_{1}\left(1+\cot ^{2} \vartheta_{1} \cos ^{2} \varphi_{1}\right)^{1 / 2}\right]}{\cos \vartheta_{1} \sin \varphi_{1}\left(1+\cot ^{2} \vartheta_{1} \cos ^{2} \varphi_{1}\right)^{1 / 2}} \\
\times \cos \left\{\frac{N}{U}\left[r_{1}\left|\sin \varphi_{1}\right|-a \sin \vartheta_{1}\left(1+\cot ^{2} \vartheta_{1} \cos ^{2} \varphi_{1}\right)\right]\right\} .
\end{aligned}
$$

The vertical displacement field in the horizontal plane $z / a=5$ is represented in figure 8 in perspective view, so as to better illustrate the shape of the isopycnal surfaces, and in figure 9 in plane view, so as to better illustrate the distribution of the wave amplitudes. The field of view has been defined after that in figures 1 and 2 of Broutman \& Rottman (2004). Two values, 1 and 1/4, of $F$ have been chosen, assumed respectively 'large' and 'small'. This choice has been made both for comparison, 1 being the value considered by Broutman \& Rottman and 1/4 the smallest value considered by Hanazaki (1988), and for illustration, 1 being as large as possible and $1 / 4$ as small as possible without preventing the distinctive influence of each representation of the sphere from being visible within the field of view. No implication must be inferred, at this stage, regarding how large or how small $F$ has to be for the respective representations to be valid approximations; this will be discussed later in $\S 8$. Accordingly, for both values of $F$, all three representations have been considered.

For the dipole, the wave field exhibits the crescent shape, associated with hyperbolic curves of constant phase, obtained theoretically by Wurtele (1957), Crapper (1959), Wu (1965), Miles (1971), Sturova (1974), Makarov \& Chashechkin (1981, 1982), Janowitz (1984), Umeki \& Kambe (1989) and Voisin (1994), among others, and observed both in the laboratory (Hopfinger et al. 1991; Bonneton et al. 1993) and in the field (Wurtele 1957; Umeki \& Kambe 1989); see also Baines (1995, § 6.1.3).

When the size of the sphere comes into play, interferences take place, which become more pronounced as $F$ becomes smaller. For weak stratification the interferences 

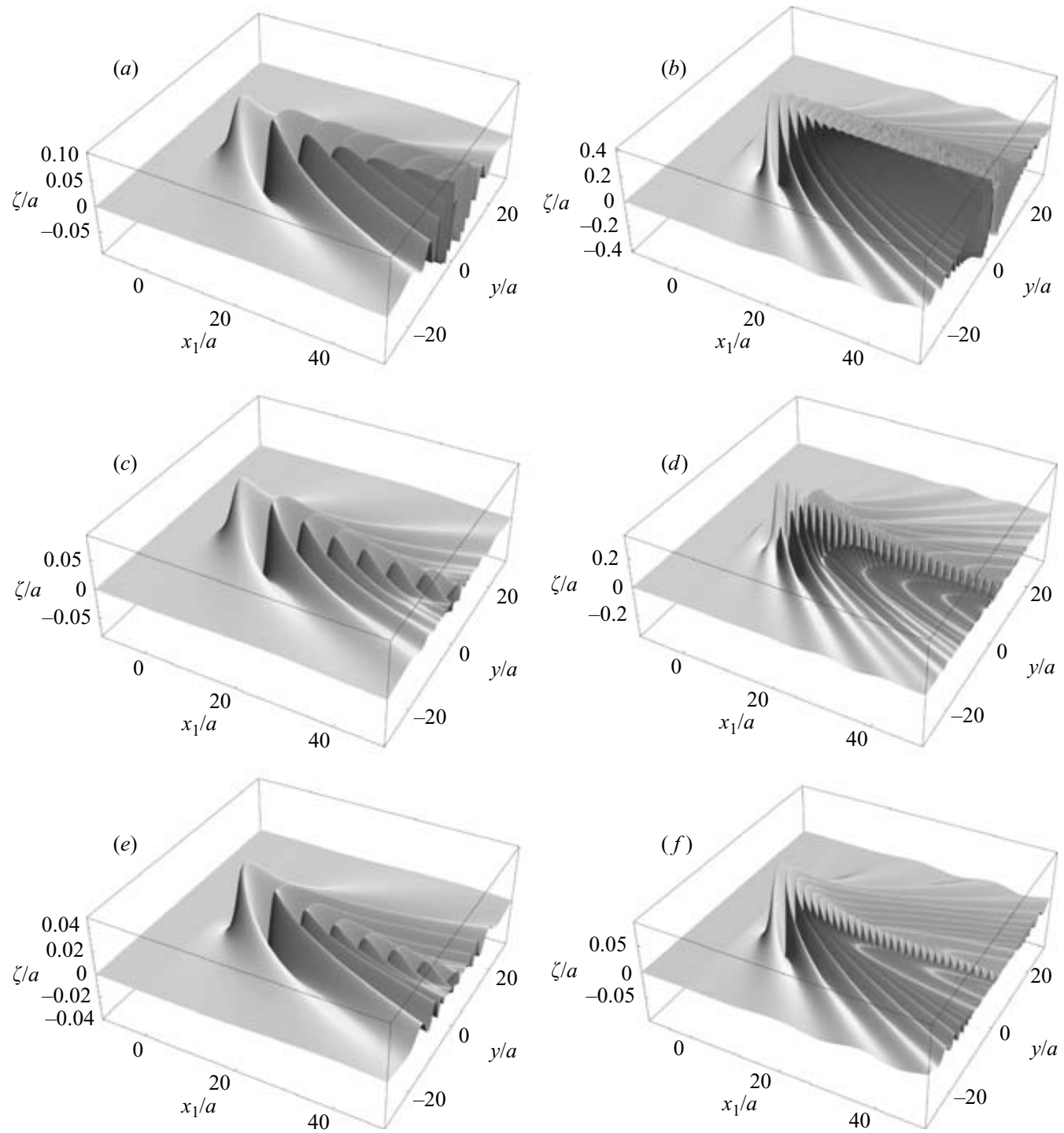

FIGURE 8. Isopycnal surface $z / a=5$ above a sphere, for $(a, b)$ the dipole $(3.1),(c, d)$ the weakly stratified model $(3.5)$ and $(e, f)$ the strongly stratified model (3.14). The internal Froude number is $F=1$ in $(a),(c)$ and $(e)$, and $F=1 / 4$ in $(b),(d)$ and $(f)$.

manifest themselves through the amplitude factor

$$
3 \frac{j_{1}(\kappa a)}{\kappa a} \quad \text { with } \quad \kappa a=\frac{1}{F}\left(1+\cot ^{2} \vartheta_{1} \cos ^{2} \varphi_{1}\right)^{1 / 2},
$$

and for strong stratification through the combination of the amplitude factor

$$
4 F^{2} \frac{J_{2}\left(\kappa_{\mathrm{h}} \ell^{\prime}\right)}{\left(\kappa_{\mathrm{h}} \ell^{\prime}\right)^{2}} \quad \text { with } \quad \kappa_{\mathrm{h}} \ell^{\prime}=\left(\frac{2}{F}\right)^{1 / 2} \cos \vartheta_{1}\left|\sin \varphi_{1}\right|\left(1+\cot ^{2} \vartheta_{1} \cos ^{2} \varphi_{1}\right)^{1 / 2},
$$

where $\ell^{\prime}=a(2 F)^{1 / 2}$, and the phase lag

$$
|m| a=\frac{1}{F} \sin \vartheta_{1}\left(1+\cot ^{2} \vartheta_{1} \cos ^{2} \varphi_{1}\right) .
$$


(a)

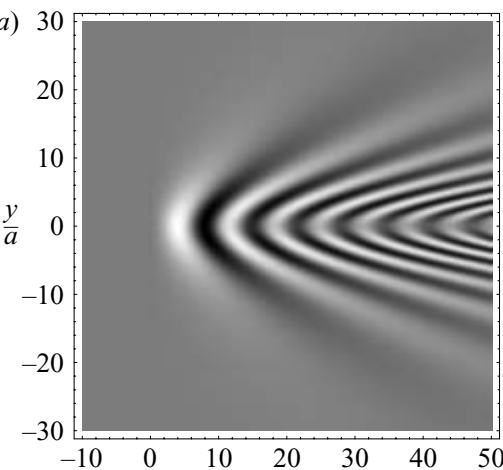

(c)

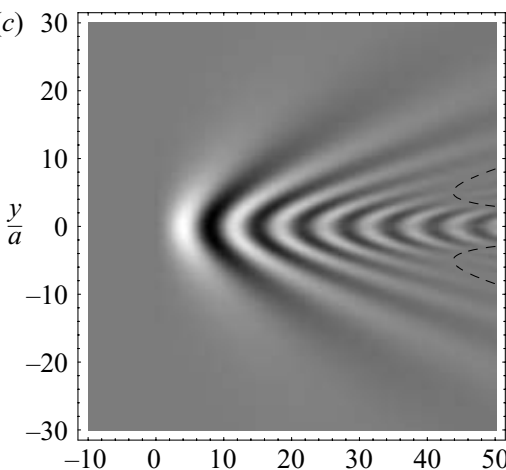

(e)

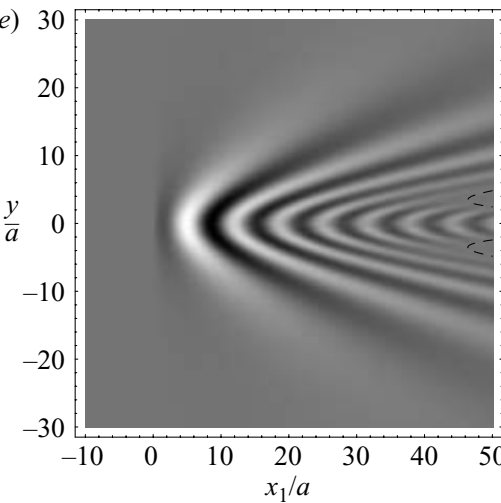

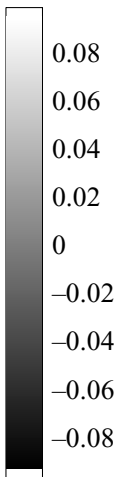

(b)

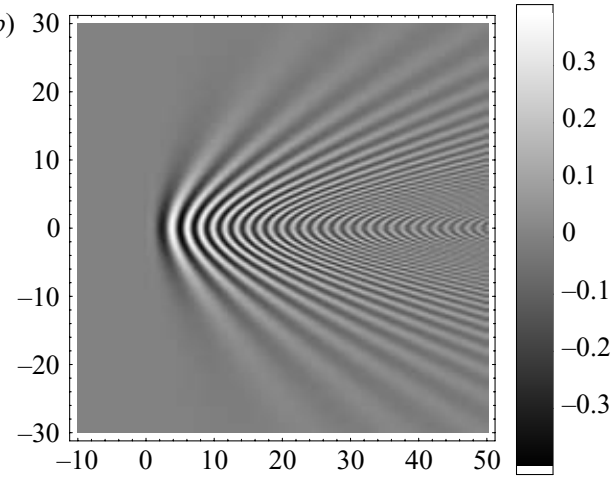

(d)

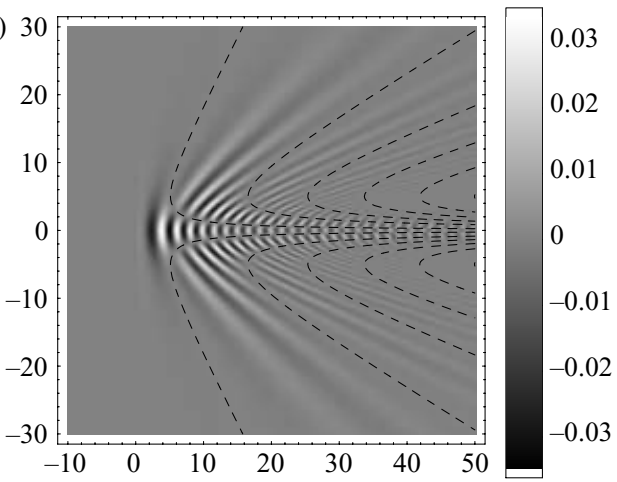

FIGURE 9. Vertical displacement $\zeta / a$ in the plane $z / a=5$ above a sphere, in the same conditions as for figure 8 . The dashed curves are fringes of destructive interference.

The two amplitude factors are represented separately in figure $10(a-d)$, in the same conditions as for figures $8(c-f)$ and $9(c-f)$.They induce an overall decrease of the wave amplitude with downstream distance $x_{1}$, together with the formation of fringes.

The fringes of destructive interference in the horizontal plane $z / a=5$ are shown in figure $9(c-f)$. They correspond to the zeros of the Bessel functions $J_{3 / 2}(\kappa a)$ for weak stratification and $J_{2}\left(\kappa_{\mathrm{h}} \ell^{\prime}\right)$ for strong stratification. In the first case the fringes are hyperbolic and the most significant amplitudes are found near the vertical planes $y=0$ and $x_{1}=0$; in the second case the most significant amplitudes are found either near the plane $y=0$, or at some distance from it outside curves close to parabolas of 

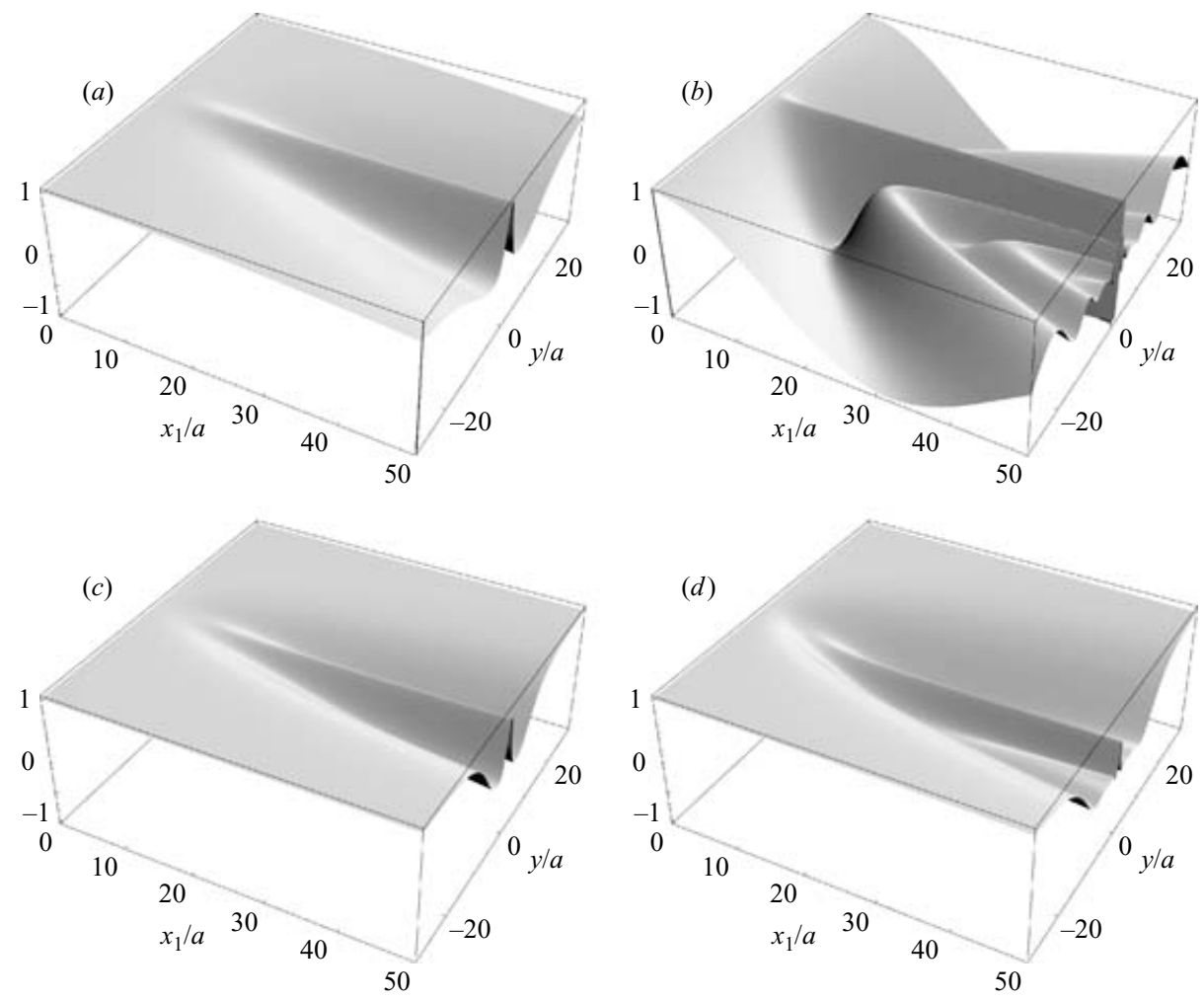

FIGURE 10. Interference factor in the plane $z / a=5$ above a sphere, for $(a, b)$ weak stratification, after normalization by $3 F j_{1}(1 / F)$, and $(c, d)$ strong stratification, after normalization by $F^{2} / 2$. The internal Froude number is $F=1$ in $(a)$ and $(c)$, and $F=1 / 4$ in $(b)$ and $(d)$.

fixed $y^{2} /\left(x_{1}|z|\right)$. The existence, in both cases, of a zone of significant amplitudes near the plane $y=0$ may explain the confinement of the waves within a fixed distance from this plane, predicted theoretically by Crapper $(1959,1962)$ and Sykes $(1978)$ for bell-shaped obstacles, and observed experimentally by Castro (1987) for a triangular ridge of finite width and numerically by Hanazaki (1988) for a sphere and Suzuki \& Kuwahara (1992) for a bell-shaped obstacle.

The assessment of these asymptotic results is twofold: both the far-field assumptions, namely $r_{1} \gg U / N$ and $r_{1} \gg a$, and the representations of the sphere, namely (3.5) at $F \gg 1$ and (3.14) at $F \ll 1$, must have their domains of validity specified.

The assessment of the far-field assumptions is made possible by Broutman \& Rottman (2004) who, in the conditions of figure 9(c), have evaluated the waves numerically based on their expression as a Fourier integral. A similar approach had been used by Sturova $(1974,1978)$ for a dipole, and by Smith $(1980)$ and Umeki \& Kambe (1989) for bell-shaped obstacles. Downstream, accounting for the minimal distance $r_{1} / a=5$ from the centre of the sphere in figures 8 and 9 , the asymptotic waves are essentially indistinguishable from the numerical ones. Upstream, the numerical solution exhibits an additional disturbance of maximum amplitude directly above or below the sphere. This upstream disturbance is the counterpart, for the threedimensional flow of a fluid of infinite depth, of the columnar disturbance observed for two-dimensional flow or for finite depth, as reviewed e.g. by Baines (1987); it cannot be predicted by the present approach, owing to the assumption of large 
propagation times. For the dipole, Voisin (1994) has presented an alternative far-field approach, based on Fourier analysis and yielding an upstream disturbance.

The assessment of the representations of the sphere is deferred until $\S 8$. For the moment, we point out the illustrative relevance of the assumed 'large' value 1 and 'small' value $1 / 4$ of $F$ : for $F>1$, the fringes of destructive interference would have moved outside the field of view in figures $8(c, e)$ and $9(c, e)$; for $F<1 / 4$, the wavelength would have become so small that the interference pattern would have been difficult to delineate in figures $8(d, f)$ and $9(d, f)$.

\section{Approximations for the strongly stratified limit}

At this point it is appropriate to go back to two approximations associated with the representation (3.14) of the sphere in strongly stratified flow, and to investigate their consistency a posteriori. For convenience, the discussion will be formulated in terms of the flow past a fixed sphere. The representation, illustrated in figure 5, is based on the idea that the waves are generated by flat cut-off obstacles, of elevation $h^{\prime}=a F$, half-length $\ell^{\prime}=a(2 F)^{1 / 2}$ and aspect ratio $\epsilon^{\prime}=(F / 2)^{1 / 2}$, lying on the dividing streamsurfaces close to the top and bottom of the sphere. The flow past these obstacles is characterized by the horizontal internal Froude number $F_{\ell^{\prime}}=(F / 2)^{1 / 2}$ and the vertical internal Froude number $F_{h^{\prime}}=1$, with $F \ll 1$.

\subsection{Linear approximation}

The first approximation, which we have used, is the linear one, found in the linearization of the boundary condition and equations of motion. Classically, for parameters $\epsilon, F_{\ell}$ and $F_{h}$, linearization is considered to require not only $\epsilon \ll 1$ but also $F_{\ell}=O(1)$, so that $F_{h} \gg 1$, corresponding to region (ii) of figure 2. Baines (1995, p. 239), in particular, indicates that the actual requirement is the smallness of the perturbation velocity $|\boldsymbol{u}|$ compared with the incoming velocity $U$, a requirement which, coupled with the order-of-magnitude estimates $\left|\boldsymbol{u}_{\mathrm{h}}\right| / U \propto 1 / F_{h}$ and $|w| / U \propto \epsilon$, yields the above conditions.

The cut-off obstacles satisfy the first condition $\epsilon^{\prime} \ll 1$ but not the following two, since $F_{\ell^{\prime}} \ll 1$ and $F_{h^{\prime}}=O(1)$, corresponding to region (iii) of figure 2 . In spite of this, the perturbation velocity is indeed small compared with the incoming velocity: at a fixed distance $r_{1}$ from the sphere comparable with its size $a$, the perturbation velocity, proportional in order of magnitude to $N$ times the vertical displacement (6.22), is such that $|\boldsymbol{u}| / U=O(F)$. Hence, linearization is allowed.

Upon closer scrutiny, Baines (1995, p. 239) is seen to assume that the motion varies horizontally on a scale $\ell$ and vertically on a scale $U / N$. By contrast, the waves (6.22) vary on the scale $U / N$ both horizontally and vertically. In figure $9(f)$, for example, the dominant horizontal variations are those of the phase, occurring on the fast scale $U / N$, while the variations of the amplitude, associated with the interference factor (6.24) and occurring on the slow scale $\ell^{\prime}$, arise as a modulation.

The explanation for this apparent paradox lies in the multilayer structure of the flow, exhibited by Greenslade (1992, 1994) and Hunt et al. (1997) and presented in $\S 3.2$ : the classical analysis by Baines $(1995$, p. 239$)$ is relevant in the top and bottom layers at the levels of the top and bottom of the sphere, respectively, where the motion has the horizontal scale $\ell^{\prime}$ and vertical scale $h^{\prime}=U / N$ of the cut-off obstacles; and the present analysis is relevant in the upper and lower layers above and below these levels, respectively, where the motion has, horizontally and vertically, the scale $U / N$ of buoyancy-induced disturbances. The waves are generated in the top and 


\begin{tabular}{|c|c|c|c|c|c|c|}
\hline \multirow[b]{2}{*}{ Region } & \multicolumn{2}{|c|}{$F \gg 1$} & \multicolumn{4}{|c|}{$F \ll 1$} \\
\hline & Inner & Outer & Middle & Top \& Bottom & Shear & Upper \& Lower \\
\hline Vertical distance & $O(1)$ & $O(F)$ & $O(1)$ & $O(F)$ & $O(F)$ & $O(1)$ \\
\hline Horizontal distance & $O(1)$ & $O(F)$ & $O(1)$ & $O\left(F^{1 / 2}\right)$ & $O(1)$ & $O(1)$ \\
\hline Perturbation velocity & $O(1)$ & $O\left(1 / F^{2}\right)$ & $O(1)$ & $O(1)$ & $O(F)$ & $O(F)$ \\
\hline Vertical displacement & $O(1)$ & $O(1 / F)$ & $O\left(F^{2}\right)$ & $O(F)$ & $O\left(F^{2}\right)$ & $O\left(F^{2}\right)$ \\
\hline
\end{tabular}

TABLE 2. Order of magnitude of the motion in each region of the fluid, for a sphere. Lengths are normalized by $a$ and velocities by $U$. For weak stratification, at $F \gg 1$, the fluid separates radially into an inner region, at $O(1)$ distance from the centre of the sphere, with motion given by (3.4), and an outer region, at $O(F)$ distance, with motion given by (6.21) in the far field. For strong stratification, at $F \ll 1$, the fluid separates vertically into top and bottom layers at $O(F)$ distance from the levels of the top and bottom of the sphere, respectively; upper and lower layers at $O(1)$ distance above and below these levels, respectively, with motion given by (6.22) in the far field; and a middle layer at $O(1)$ distance in between, with motion given by $(2.15 b)$ and $(3.7 b)$. The top and bottom layers separate in turn horizontally into top and bottom regions, at $O\left(F^{1 / 2}\right)$ distance from the top and bottom, respectively, and shear layers, at $O(1)$ distance. The shear layers have the same dynamics as the upper and lower layers, except with respect to the hydrostatic approximation; hence, for simplicity, in the rest of the paper they have been omitted and classed implicitly alongside the upper and lower layers.

bottom layers with large amplitudes, and propagate in the upper and lower layers with small amplitudes. Table 2 indicates, for both strongly and weakly stratified flows, the different regions of the fluid and the order of magnitude of the motion in each region.

\subsection{Hydrostatic approximation}

The second approximation, which we have not used, is the hydrostatic one, presented in the Appendix. The drag coefficient (5.32) becomes, with the assumption $\xi=U|k| / N \ll 1$,

$$
C_{D}=\frac{32}{\pi} F^{2} \int_{0}^{\infty} \frac{\mathrm{d} \xi}{\xi^{2}} \int_{1}^{\infty} \frac{\mathrm{d} \eta}{\eta^{4}\left(\eta^{2}-1\right)^{1 / 2}} J_{2}^{2}\left[\xi \eta\left(\frac{2}{F}\right)^{1 / 2}\right],
$$

which is evaluated exactly as

$$
C_{D}=\frac{2^{11 / 2}}{15 \pi} F^{3 / 2},
$$

namely as the leading-order term (5.46) of the expansion (5.45) of the non-hydrostatic drag coefficient at small $F$. The vertical displacement (6.22) becomes, with the assumption $|z| \ll r_{\mathrm{h}_{1}}$,

$$
\zeta(x, t) \sim 2 \frac{U^{2}}{N^{2}\left(x_{1}^{2}+y^{2}\right)^{1 / 2}} \frac{y^{2}}{x_{1} z} J_{2}\left[\left(2 \frac{N a}{U}\right)^{1 / 2} \frac{x_{1} z}{y^{2}}\right] \cos \left[\frac{N}{U}(|z|-a)\left(1+\frac{x_{1}^{2}}{y^{2}}\right)^{1 / 2}\right] .
$$

Figure 11 represents the associated displacement field in the horizontal plane $z / a=5$, together with the amplitude factor

$$
4 F^{2} \frac{J_{2}\left(\kappa_{\mathrm{h}} \ell^{\prime}\right)}{\left(\kappa_{\mathrm{h}} \ell^{\prime}\right)^{2}} \quad \text { with } \quad \kappa_{\mathrm{h}} \ell^{\prime}=\left(\frac{2}{F}\right)^{1 / 2} \frac{x_{1}|z|}{y^{2}} .
$$



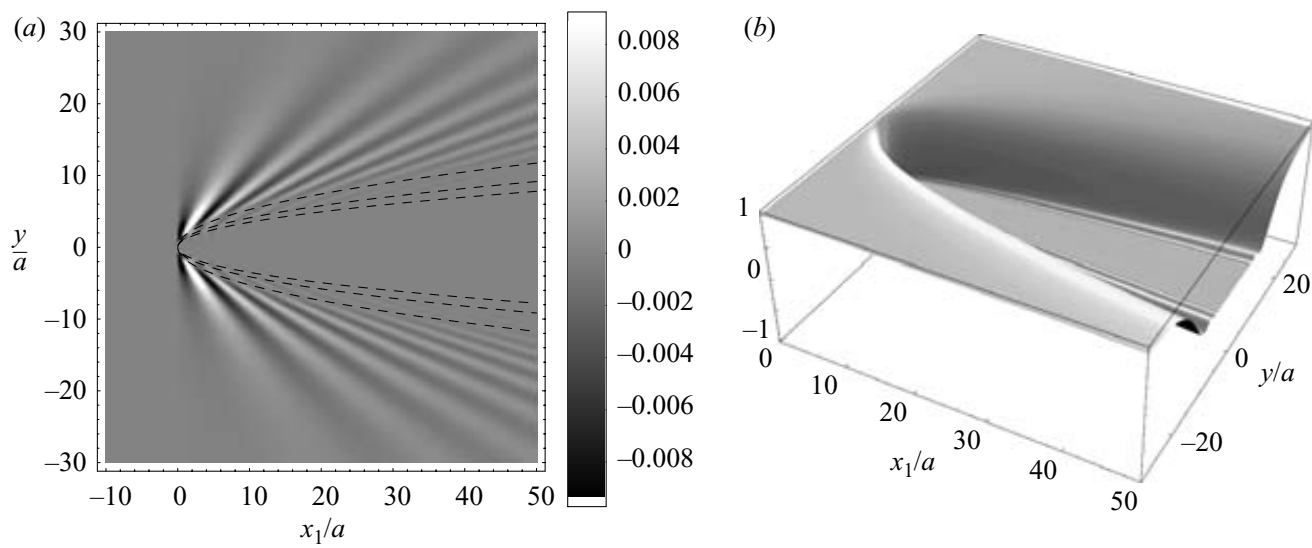

FiguRE 11. Hydrostatic approximation, for strongly stratified flow past a sphere, of $(a)$ the vertical displacement $\zeta / a$ and $(b)$ the interference factor normalized by $F^{2} / 2$, in the plane $z / a=5$ at $F=1 / 4$. The dashed curves are the first three fringes of destructive interference.

Consistently with Smith (1980) and Baines (1995, §6.1.4), the interference fringes are turned into parabolae. For destructive interference, they have the equation

$$
y^{2}=\left(\frac{2}{F}\right)^{1 / 2} \frac{x_{1}|z|}{j_{2, n}}
$$

with $n=1,2,3, \ldots$, and $j_{2, n}$ the zeros of the Bessel function $J_{2}(x)$. The first three such fringes are shown in figure 11(a); as $n$ increases further, they become rapidly indistinguishable from one another and accumulate near the plane $y=0$.

Classically, for parameters $\epsilon, F_{\ell}$ and $F_{h}$, the hydrostatic approximation is considered to require both $F_{\ell} \ll 1$ and $F_{h}=O(1)$, so that $\epsilon \ll 1$, corresponding to region (iii) of figure 2. The cut-off obstacles satisfy all three conditions. However, comparison of the hydrostatic waves in figures $11(a)$ and 11(b) with the non-hydrostatic waves in figures $9(f)$ and $10(d)$, respectively, shows that the hydrostatic approximation is valid only outside, say, the first fringe of destructive interference; inside this fringe, the amplitudes are underestimated close to the plane $y=0$ and overestimated close to the plane $x_{1}=0$. From similar comparisons for a plane-mounted bell-shaped obstacle, Smith (1980) has observed, theoretically, that the hydrostatic approximation describes satisfactorily the motion of the fluid directly above the obstacle, and the first crest and trough of the waves immediately behind it, but fails to account for the trailing waves further downstream, while Miranda \& James (1992) have observed, numerically, that the area of validity of the hydrostatic approximation is displaced further away on either side of the plane $y=0$ as the vertical distance $z$ from the obstacle increases. Both observations are consistent with a criterion of validity involving the first fringe of destructive interference.

This apparent paradox illustrates the deceptive nature of a priori simplifications to a wave field, based on the comparison of fixed space and time scales: for dispersive waves, the appropriate scales are the inverse wavenumber and frequency, respectively, which vary spatially and temporally through the wave field; for them, the only legitimate approach consists in looking for simplifications based on the spectrum of the forcing, which determines the wavenumbers and frequencies actually radiated. In particular, the spectrum (3.15) includes the Bessel function $J_{2}\left(\kappa_{\mathrm{h}} \ell^{\prime}\right)$, implying that only the wavenumbers such that $\kappa_{\mathrm{h}} \ell^{\prime} \lesssim 1$ contribute significantly to the radiation. 
For the drag coefficient (5.32), the condition $\kappa_{\mathrm{h}} \ell^{\prime} \lesssim 1$ is expressed in terms of the variables $\xi=U|k| / N$ and $\eta=\kappa_{\mathrm{h}} /|k|$ as

$$
\xi \eta \lesssim\left(\frac{F}{2}\right)^{1 / 2}
$$

with $\eta>1$. Hence, for $F \ll 1$, the hydrostatic assumption $\xi \ll 1$ follows naturally. For the vertical displacement (6.22), however, the condition $\kappa_{\mathrm{h}} \ell^{\prime} \lesssim 1$ is expressed in terms of the position $\left(x_{1}, y, z\right)$ as

$$
\frac{x_{1}|z|}{y^{2}+z^{2}}\left[\frac{1+x_{1}^{2} y^{2} /\left(y^{2}+z^{2}\right)^{2}}{1+x_{1}^{2} /\left(y^{2}+z^{2}\right)}\right]^{1 / 2} \lesssim\left(\frac{F}{2}\right)^{1 / 2} .
$$

Hence, for $F \ll 1$, the hydrostatic assumption $|z| \ll\left(x_{1}^{2}+y^{2}\right)^{1 / 2}$ is just one among several ways to ensure that it is satisfied. Replacing, as a first approximation, (7.7) by its hydrostatic form, namely

$$
y^{2} \gtrsim\left(\frac{2}{F}\right)^{1 / 2} x_{1}|z|,
$$

we recover the above criterion involving the parabolae (7.5).

Again, the explanation for the apparent paradox is found within the multilayer structure of the flow, exhibited by Greenslade $(1992,1994)$ and Hunt et al. (1997) and presented in $\S 3.2$ : the waves are hydrostatic in the top and bottom layers, whose small thickness implies quasi-horizontal propagation, and non-hydrostatic in the upper and lower layers. Taking into account the further horizontal decomposition of the top and bottom layers into top and bottom regions close to the top and bottom of the sphere, respectively, and shear layers away from them, as indicated in table 2, we anticipate that the motion is nonlinear hydrostatic in the top and bottom regions, linear hydrostatic in the shear layers, and linear non-hydrostatic in the upper and lower layers.

\section{Discussion and conclusions}

Based on asymptotic analysis of the horizontal flow at velocity $U$ of a stratified fluid of buoyancy frequency $N$ past a sphere of radius $a$, two distinct representations of the sphere as a source of lee waves have been proposed in $\S 3$, appropriate for either weak or strong stratification and corresponding to either large or small internal Froude number $F=U /(\mathrm{Na})$, respectively. In the following, vertical displacements are normalized by $a$ and velocities by $U$, and the orders of magnitude are taken from table 2.

For $F \gg 1$, the fluid separates into two regions radially: at distances $r_{1}$ from the centre of the sphere of the order of $a$, an inner region where the flow is essentially unaffected by the stratification and reduces, to $O(1)$, to the three-dimensional irrotational flow (3.4), illustrated in figure 3; at distances $r_{1}$ of the order of the scale $U / N$ of buoyancy-induced motion, an outer region with small-amplitude lee waves of $O(1 / F)$ vertical displacement (6.21) in the far field, illustrated in figures $8(c)$ and $9(c)$. The waves propagate in the outer region, with small amplitudes, and are generated by the vertical motion in the inner region. This leads to the identification of the source (3.5) of the inner flow as the source of the waves.

For $F \ll 1$, the fluid separates into five layers vertically: at distances $|z|$ from the centre of the sphere smaller than $(1-F) a$, a middle layer where the flow is essentially 


\begin{tabular}{lcccc} 
Obstacle & \multicolumn{1}{c}{ Profile } & $\alpha$ & $\beta$ & $B$ \\
Semi-ellipsoid & $z=h\left(1-\frac{x_{1}^{2}+y^{2}}{\ell^{2}}\right)^{1 / 2}$ & $\frac{\pi}{4}$ & 1 & $\frac{32 \sqrt{2}}{15 \pi} \approx 0.96$ \\
Gaussian hill & $z=h \exp \left(-\frac{x_{1}^{2}+y^{2}}{\ell^{2}}\right)$ & $\frac{\sqrt{ } \pi}{2}$ & 2 & $\frac{16}{15 \sqrt{ } \pi} \approx 0.60$ \\
Witch of Agnesi & $z=h\left(1+\frac{x_{1}^{2}+y^{2}}{\ell^{2}}\right)^{-3 / 2}$ & 1 & 3 & $\frac{8 \sqrt{ } 2}{15 \sqrt{3}} \approx 0.43$
\end{tabular}

TABLE 3. Wave drag coefficient $C_{D}$ at small $F$ for typical obstacles of revolution of vertical axis, height $h$, half-length $\ell$ and locally paraboloidal shape at the summit. $\alpha$ is the ratio of the area cross-section of the obstacle to the reference area $2 h \ell$, and $\beta$ is the scaled curvature at the summit defined according to (3.9). To leading order, $C_{D} \sim B F^{3 / 2}$ with $B$ an $O(1)$ positive constant.

horizontal, around the sphere rather than over or under it, and reduces, to $O(1)$, to the two-dimensional horizontal irrotational flow $(2.15 b)$ with associated $O\left(F^{2}\right)$ vertical displacement (3.7b), illustrated in figure 4; at distances $|z|$ between $(1-F) a$ and $a$, top and bottom layers with $O(F)$ vertical displacement; at distances $|z|$ larger than $a$, upper and lower layers with small-amplitude lee waves of $O\left(F^{2}\right)$ vertical displacement (6.22) in the far field, illustrated in figures $8(f)$ and $9(f)$. The waves propagate in the upper and lower layers, with small amplitudes, and are generated by the vertical motion in the top and bottom layers, respectively. This leads to the identification of the top and bottom portions of the sphere, lying above and below the dividing streamsurfaces $|z|=(1-F) a$ separating the top and bottom layers from the middle layer, respectively, as the source of the waves; these portions, illustrated in figure 5, act as flat cut-off obstacles of representation (3.14).

The analysis has been validated by comparison of the prediction of the wave drag in $\S 5$ with the interpretation by Greenslade (2000), recalled and extended in $\S 1$, of the available measurements of the drag on a sphere. For $F \ll 1$, not only has the variation of the wave drag coefficient $C_{D}$ as $F^{3 / 2}$ been recovered, but also the multiplier $B$ in front of the variation has been predicted analytically as $(32 \sqrt{ } 2) /(15 \pi) \approx 0.96$. This result may easily be generalized: a plane-mounted obstacle of revolution of vertical axis, height $h$, half-length $\ell$ and locally paraboloidal shape (3.9) at the summit generates waves as the source (3.12) and has the wave drag coefficient

$$
C_{D} \sim \frac{8}{15 \alpha}\left(\frac{2}{\beta}\right)^{1 / 2} F^{3 / 2},
$$

with $\alpha$ the ratio of the area cross-section to the reference area $2 h \ell$, and $\beta$ the scaled curvature at the summit. As a consequence, not only does $C_{D}$ vary as $F^{3 / 2}$ irrespective of the exact shape, but also $B$ is independent of the aspect ratio $\epsilon$. Table 3 illustrates this for three typical obstacles, emphasizing in particular that, in this regime, any semi-ellipsoid has the same $C_{D}$ as the hemisphere.

That being said, the intricacy of the mathematical procedure, involving MellinBarnes integration in $\S 5$, must not hide the essentially phenomenological nature of the two representations of the sphere. For $F \gg 1$, the representation (3.5) is based on the radial separation of the fluid into two regions, respectively inner and outer, suggesting the application of the method of matched asymptotic expansions. This method has been mentioned by Miles \& Huppert (1969) for two-dimensional 
obstacles and Miles (1971) for three-dimensional obstacles, but so far it has only been implemented by Baines \& Grimshaw (1979) for two-dimensional obstacles. For $F \ll 1$, the representation (3.14) is based on the vertical separation of the fluid into five layers, in connection with the formation of dividing streamsurfaces. In each layer the dominant mechanisms have been identified by Greenslade $(1992,1994)$ and Hunt et al. (1997), and the equations of motion have been written, but these equations remain to be solved and their solutions matched. Only such rigorous mathematical derivation will be able to provide a definitive basis for the ideas developed in the present paper.

Similarly, the derivation of expansions of the wave drag coefficient to all orders in $\S 5$ may seem spurious, given the leading-order nature of the underlying representations of the sphere. It is believed, however, that the ability to carry the expansions to all orders will prove determinant when implementing proper asymptotic matching between the motions in different regions of the fluid.

A more stringent validation is the comparison of the prediction of the wave field in $\S 6$ with its experimental and numerical determinations. Unfortunately, such determinations are scarce in the literature for the sphere.

Early experiments have focused on schlieren visualization of the surfaces of constant phase (Peat \& Stevenson 1975; Makarov \& Chashechkin 1981, 1982; Chashechkin 1989), sometimes complemented with point conductimetric measurement of the amplitude (Makarov \& Chashechkin 1981, 1982). Later, laser-induced fluorescence has provided a spatial picture of this amplitude (Hopfinger et al. 1991; Bonneton et al. 1993; Dupont \& Voisin 1996). Figure 3 of Bonneton et al., in particular, shows good overall agreement between the visualization of the vertical displacement in the horizontal plane $z / a=3$ and its prediction (6.20) for the dipole (3.1), at $F=3.2$ large enough for the effect of the size of the sphere to be negligible within the field of view. It is only very recently, however, that particle image velocimetry has allowed the picture to become quantitative. Figures 3 and 4 of Rottman et al. (2004) exhibit, at $F=1$ and 2 in the horizontal plane $z / a=4$, the consistency of the visualization of the vertical displacement with its prediction (6.21) for the weakly stratified model (3.5), after accounting for the formation of vertical modes due to the finite depth of the experimental tank. More elaborate consideration of this depth, through the inclusion of evanescent modes, is required before definitive conclusions can be drawn.

The only available numerical data are those of Hanazaki (1988). Figure 12(a,b) represents, in the same conditions as his figure $3(c, f)($ iii), respectively, the predicted isopycnal lines.At $F=1$, the $O(1)$ inner flow (3.4) has been superposed everywhere with the $O(1 / F)$ far-field outer waves $(6.21)$; at $F=1 / 4$, the $O\left(F^{2}\right)$ flow $(3.7 b)$ in the middle layer has been complemented with the $O\left(F^{2}\right)$ far-field waves (6.22) everywhere in the upper and lower layers. Apart from an overestimation of the wavelength in the theory, already noted by Hanazaki, the comparison exhibits reasonably good agreement between theory and simulation a few radii away from the sphere. Closer to the sphere, discrepancies are observed including unphysical large amplitudes and crossings of the isopycnals in the theory: this exhibits the futility of applying a far-field result in the near field, together with the need for proper solution of the equations of motion and matching of the solutions in the different regions of the fluid.

As a rule, based on the comparison of the measured and predicted drag coefficients in figure 1 , we may expect the large- $F$ theory to be valid, say, at $F \gtrsim 1$, and the small$F$ theory, say, at the same time as Sheppard's criterion for the dividing streamsurfaces, namely for the sphere at $F \lesssim 0.4$. From a similar comparison, Greenslade (2000) has 
(a)

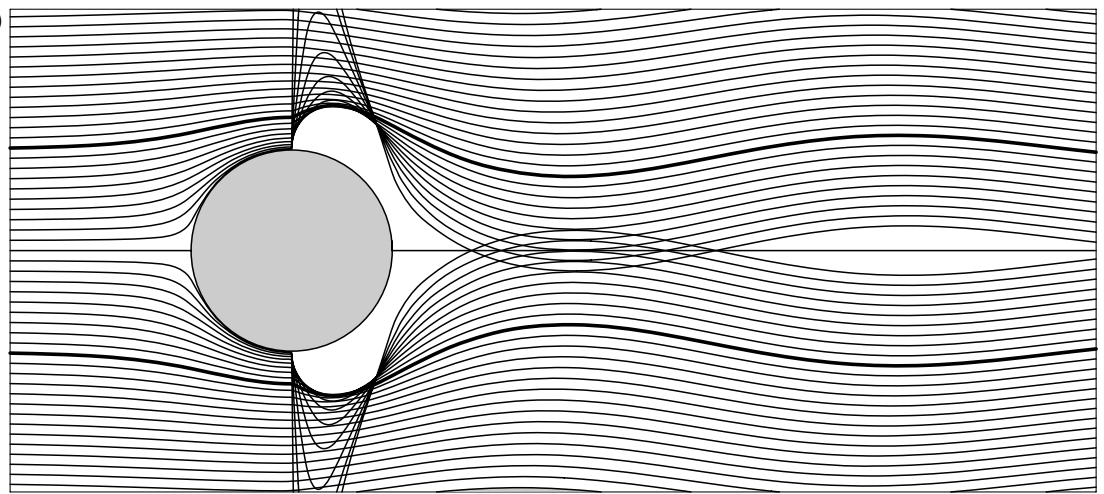

(b)

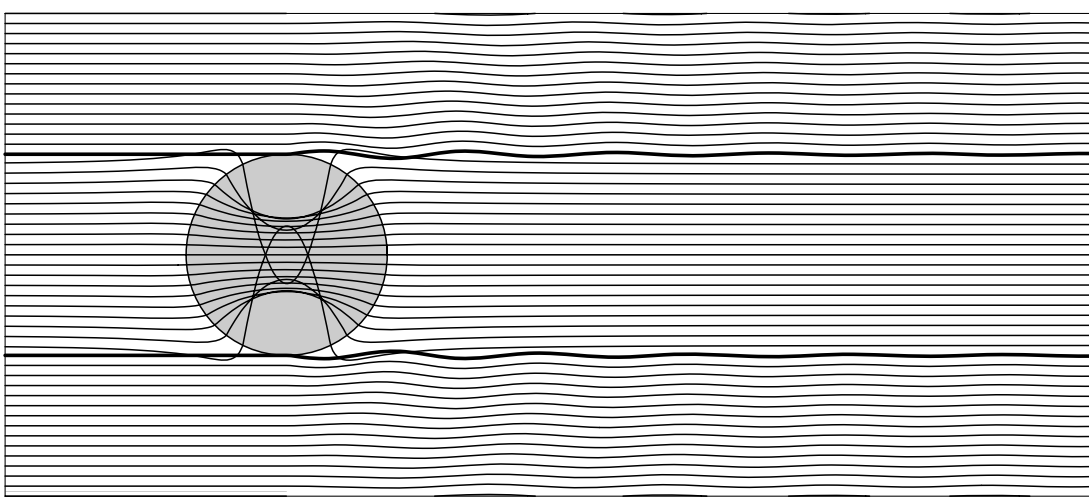

FIGURE 12. Isopycnals for the flow past a sphere, using $(a)$ the weakly stratified model of $\S 3.1$ at $F=1$ and $(b)$ the strongly stratified model of $\S 3.2$ at $F=1 / 4$. The isopycnals are drawn in the vertical plane $y=0$ and on the sphere surface. They are constructed by superposing in (a) the $O(1 / F)$ waves (6.21) with the $O(1)$ flow (3.4), and in $(b)$ the $O\left(F^{2}\right)$ waves $(6.22)$ for $|z|>a$ with the $O\left(F^{2}\right)$ flow $(3.7 b)$ for $|z|<a$. The thick lines originate from the levels of the top and bottom of the sphere at infinity upstream.

introduced the idea that, apart from a narrow intermediate range $0.8<F<1.1$, the large- $F$ theory is valid for $F>1.1$ and the small- $F$ theory for $F<0.8$.

Finally, it must be pointed out that the analysis has considered the wave contribution to the drag exclusively, and omitted the wake contribution entirely. In practice, consistently with Greenslade's (2000) model (1.6), this latter contribution is dominant at small $F$. The formation and shedding of vorticity in the lee of obstacles in strongly stratified flow has been an area of active research for more than two decades, with its origin in the experiments of Brighton (1978), Hunt \& Snyder (1980) and Castro et al. (1983), and in the numerical simulations of Smolarkiewicz \& Rotunno (1989) and Rotunno \& Smolarkiewicz (1991); for recent advances and detailed bibliographies, see the numerical simulations of Schär \& Durran (1997), Rotunno, Grubišić \& Smolarkiewicz (1999), Vosper (2000) and Castro et al. (2001), and the experiments of Vosper et al. (1999) and Castro et al. (2001).

Similarly, at large $F$, say for a sphere $F \gtrsim 4$, the steady lee waves produced by the flow in the immediate vicinity of the obstacle give way to so-called 'random' internal waves produced by the motion and collapse of the coherent structures of the wake. This phenomenon has been observed experimentally for a slender body by Gilreath \& Brandt (1985), and investigated more thoroughly for a sphere by Sysoeva \& 
Chashechkin (1986, 1991), Hopfinger et al. (1991), Bonneton et al. (1993, 1996), Lin, Boyer \& Fernando (1993) and Rottman et al. (2004), and for a bell-shaped obstacle by Dupont, Kadri \& Chomaz (2001). Wake-generated internal waves may also be seen in the numerical simulations of Gourlay et al. (2001). The contribution of these waves to the drag need not be considered explicitly, as it is already included in the wake contribution. For the waves themselves, when the advection of the coherent structures before their collapse is negligible, the forcing may be modelled as a source fixed with respect to the obstacle and of strength oscillating at the frequency of vortex shedding, a model mentioned first by Gilreath \& Brandt (1985), developed later by Voisin (1995) and Dupont \& Voisin (1996), and applied recently by Rottman et al. (2004).

The author would like to thank Dr Michael Greenslade for advice and encouragement, and for generously sharing his digitization of the data of Mason and Lofquist \& Purtell. Professors Valentin Gorodtsov, Yuli Chashechkin, Drs Olga Shishkina, Evgeny Ermanyuk and Dave Broutman are thanked for stimulating conversations and correspondence.

\section{Appendix. Hydrostatic lee waves}

The hydrostatic approximation lies in the omission of the vertical acceleration in the Euler equation (4.1a). It is based on the assumption of low frequency $|\omega| \ll N$ or, by the dispersion relation $|\omega|=N \kappa_{\mathrm{h}} / \kappa$, quasi-horizontal propagation, namely quasivertical wavenumber vector, such that $\kappa_{\mathrm{h}} \ll|m|$, and quasi-horizontal group velocity. The wave equation (4.3) simplifies to

$$
\left(\frac{\partial^{2}}{\partial t^{2}} \frac{\partial^{2}}{\partial z^{2}}+N^{2} \nabla_{\mathrm{h}}^{2}\right) \chi=q
$$

with

$$
\rho=\rho_{0} \frac{N^{2}}{g} \frac{\partial^{2}}{\partial t \partial z} \chi, \quad p=-\rho_{0} N^{2} \frac{\partial}{\partial t} \chi, \quad \boldsymbol{u}=\left(\frac{\partial^{2}}{\partial t^{2}} \frac{\partial}{\partial z} \boldsymbol{e}_{z}+N^{2} \nabla_{\mathrm{h}}\right) \chi .
$$

For horizontal motion of a body at velocity $U$, or for horizontal flow at velocity $U$ past an obstacle, another formulation of the assumption is, by the Doppler relation $\omega=-U k$, the condition $|k| \ll N / U$ that the waves are long in the streamwise direction. The wave drag (5.5) simplifies to

$$
D=\frac{\rho_{0} N}{16 \pi^{2} U} \sum_{ \pm} \int_{-\infty}^{\infty} \mathrm{d} k \int_{-\infty}^{\infty} \mathrm{d} l \frac{\left|q_{0}\left(\boldsymbol{k}_{ \pm}\right)\right|^{2}}{\left(k^{2}+l^{2}\right)^{1 / 2}}
$$

with

$$
\boldsymbol{k}_{ \pm}=\left[k, l, \pm \frac{N}{U}\left(1+\frac{l^{2}}{k^{2}}\right)^{1 / 2}\right]
$$

Similarly, by the assumption $|z| \ll r_{\mathrm{h}_{1}}$ of quasi-horizontal propagation, the vertical displacement (6.19) simplifies to

$$
\zeta(\boldsymbol{x}, t) \sim-\frac{\operatorname{sign} z}{2 \pi U|y|} \operatorname{Im}\left\{q_{0}\left(\boldsymbol{k}, t_{\mathrm{s}}\right) \exp \left[-\mathrm{i} \frac{N}{U}|z|\left(1+\frac{x_{1}^{2}}{y^{2}}\right)^{1 / 2}\right]\right\},
$$

with

$$
\boldsymbol{k}=\frac{N}{U}\left(1+\frac{x_{1}^{2}}{y^{2}}\right)^{1 / 2}\left[-\frac{x_{1}|z|}{x_{1}^{2}+y^{2}}, \frac{x_{1}^{2}|z|}{y\left(x_{1}^{2}+y^{2}\right)},-\operatorname{sign} z\right]
$$




\section{REFERENCES}

Aksenov, A. V., Gorodtsov, V. A. \& Sturova, I. V. 1986 Modeling of the flow of a stratified ideal incompressible fluid around a cylinder. Preprint No. 282, Institute for Problems in Mechanics, Russian Academy of Sciences, Moscow (in Russian).

AkYLAS, T. R. \& Davis, K. S. 2001 Three-dimensional aspects of nonlinear stratified flow over topography near the hydrostatic limit. J. Fluid Mech. 428, 81-105.

BaINes, P. G. 1987 Upstream blocking and airflow over mountains. Annu. Rev. Fluid Mech. 19, 75-97.

BaInes, P. G. 1995 Topographic Effects in Stratified Flows. Cambridge University Press.

BAINES, P. G. \& GRIMSHAw, R. H. J. 1979 Stratified flow over finite obstacles with weak stratification. Geophys. Astrophys. Fluid Dyn. 13, 317-334.

Baines, P. G. \& Sмith, R. B. 1993 Upstream stagnation points in stratified flow past obstacles. Dyn. Atmos. Oceans 18, 105-113.

BARnes, E. W. 1906 The asymptotic expansion of integral functions defined by Taylor's series. Phil. Trans. R. Soc. Lond. A 206, 249-297.

BARNES, E. W. 1907 The asymptotic expansion of integral functions defined by generalised hypergeometric series. Proc. Lond. Math. Soc. (2) 5, 59-116.

BARnes, E. W. 1908 On functions defined by simple types of hypergeometric series. Trans. Camb. Phil. Soc. 20, 253-279.

Batchelor, G. K. 1967 An Introduction to Fluid Dynamics. Cambridge University Press.

Bleistein, N. \& Handelsman, R. A. 1986 Asymptotic Expansions of Integrals, 2nd edn. Dover.

Blumen, W. 1965 A random model of momentum flux by mountain waves. Geofys. Publ. 26(2), $1-33$.

Blumen, W. \& McGregor, C. D. 1976 Wave drag by three-dimensional mountain lee-waves in nonplanar shear flow. Tellus 28, 287-298.

Bonneton, P., Chomaz, J.-M. \& Hopfinger, E. J. 1993 Internal waves produced by the turbulent wake of a sphere moving horizontally in a stratified fluid. J. Fluid Mech. 254, 23-40.

Bonneton, P., Chomaz, J.-M., Hopfinger, E. J. \& Perrier, M. 1996 The structure of the turbulent wake and the random internal wave field generated by a moving sphere in a stratified fluid. Dyn. Atmos. Oceans 23, 299-308.

Bretherton, F. P. 1967 The time-dependent motion due to a cylinder moving in an unbounded rotating or stratified fluid. J. Fluid Mech. 28, 545-570.

Brighton, P. W. M. 1978 Strongly stratified flow past three-dimensional obstacles. Q. J. R. Met. Soc. 104, 289-307.

Broutman, D. \& Rottman, J. W. 2004 A simplified Fourier method for computing the internal wavefield generated by an oscillating source in a horizontally moving, depth-dependent background. Phys. Fluids 16, 3682-3689.

CAstro, I. P. 1987 A note on lee wave structures in stratified flow over three-dimensional obstacles. Tellus A 39, 72-81.

Castro, I. P., Snyder, W. H. \& Marsh, G. L. 1983 Stratified flow over three-dimensional ridges. J. Fluid Mech. 135, 261-282.

Castro, I., Vosper, S., Paisley, M. \& Hayden, P. 2001 Vortex shedding behind tapered obstacles in neutral and stratified flow. Dyn. Atmos. Oceans 34, 145-163.

Chashechin, Yu. D. 1989 Hydrodynamics of a sphere in a stratified fluid. Fluid Dyn. 24, 1-7. [Transl. from Izv. Akad. Nauk SSSR Mekh. Zhidk. Gaza (1), 3-9.]

Chomaz, J.-M., Bonneton, P., Butet, A., Perrier, M. \& Hopfinger, E. J. 1992 Froude number dependence of the flow separation line on a sphere towed in a stratified fluid. Phys. Fluids A 4, 254-258.

Chomaz, J.-M., Bonneton, P. \& Hopfinger, E. J. 1993 The structure of the near wake of a sphere moving horizontally in a stratified fluid. J. Fluid Mech. 254, 1-21.

Crapper, G. D. 1959 A three-dimensional solution for waves in the lee of mountains. J. Fluid Mech. 6, 51-76.

Crapper, G. D. 1962 Waves in the lee of a mountain with elliptical contours. Phil. Trans. R. Soc. Lond. A 254, 601-623. 
Dokuchaev, V. P. \& Dolina, I. S. 1977 Radiation of internal waves by sources in an exponentially stratified fluid. Izv. Atmos. Ocean. Phys. 13, 444-449. [Transl. from Izv. Akad. Nauk SSSR Fiz. Atmos. Okeana 13, 655-663.]

Drazin, P. G. 1961 On the steady flow of a fluid of variable density past an obstacle. Tellus 13, 239-251.

Drazin, P. G. \& Su, C. H. 1975 A note on long-wave theory of airflow over a mountain. J. Atmos. Sci. 32, 437-439.

Dupont, P., KADRI, Y. \& ChOMAZ, J.-M. 2001 Internal waves generated by the wake of Gaussian hills. Phys. Fluids 13, 3223-3233.

Dupont, P. \& Voisin, B. 1996 Internal waves generated by a translating and oscillating sphere. Dyn. Atmos. Oceans 23, 289-298.

EAmes, I. \& Hunt, J. C. R. 1997 Inviscid flow around bodies moving in weak density gradients without buoyancy effects. J. Fluid Mech. 353, 331-355.

EgAN, B. A. 1984 Transport and diffusion in complex terrain (review). Boundary-Layer Met. 30, $3-28$.

Gilreath, H. E. \& Brandt, A. 1985 Experiments on the generation of internal waves in a stratified fluid. AIAA J. 23, 693-700.

Gorodtsov, V. A. 1980 Radiation of internal waves during vertical motion of a body through a nonuniform liquid. J. Engng Phys. 39, 1062-1065. [Transl. from Inzh.-Fiz. Zh. 39, 619-623.]

Gorodtsov, V. A., Reznik, S. N. \& Stepanyants, Yu. A. 1997 Radiative forces acting on point sources moving in a stratified fluid. J. Expl Theor. Phys. 85, 276-284. [Transl. from Zh. Éksp. Teor. Fiz. 112, 507-523.]

Gorodtsov, V. A. \& Teodorovich, É. V. 1980 On the generation of internal waves in the presence of uniform straight-line motion of local and nonlocal sources. Izv. Atmos. Ocean. Phys. 16, 699-704. [Transl. from Izv. Akad. Nauk SSSR Fiz. Atmos. Okeana 16, 954-961.]

Gorodtsov, V. A. \& TeOdorovich, É. V. 1981 Two-dimensional problem for internal waves generated by moving singular sources. Fluid Dyn. 16, 219-224. [Transl. from Izv. Akad. Nauk SSSR Mekh. Zhidk. Gaza (2), 77-83.]

Gorodtsov, V. A. \& Teodorovich, É. V. 1982 Study of internal waves in the case of rapid horizontal motion of cylinders and spheres. Fluid Dyn. 17, 893-898. [Transl. from Izv. Akad. Nauk SSSR Mekh. Zhidk. Gaza (6), 94-100.]

Gorodtsov, V. A. \& TeOdorovich, É. V. 1983 Radiation of internal waves by periodically moving sources. J. Appl. Mech. Tech. Phys. 24, 521-526. [Transl. from Zh. Prikl. Mekh. Tekh. Fiz. (4), 81-87.]

Gourlay, M. J., Arendt, S. C., Fritts, D. C. \& Werne, J. 2001 Numerical modeling of initially turbulent wakes with net momentum. Phys. Fluids 13, 3783-3802.

Greenslade, M. D. 1992 Strongly stratified airflow over and around mountains. Ph.D. thesis, University of Leeds.

Greenslade, M. D. 1994 Strongly stratified airflow over and around mountains. In Stably Stratified Flows: Flow and Dispersion over Topography (ed. I. P. Castro \& N. J. Rockliff), pp. 25-37. Oxford University Press.

Greenslade, M. D. 2000 Drag on a sphere moving horizontally in a stratified fluid. J. Fluid Mech. 418, 339-350.

Grimshaw, R. 1969 Slow time-dependent motion of a hemisphere in a stratified fluid. Mathematika 16, 231-248.

HANAZAKI, H. 1988 A numerical study of three-dimensional stratified flow past a sphere. J. Fluid Mech. 192, 393-419.

Hawthorne, W. R. \& Martin, M. E. 1955 The effect of density gradient and shear on the flow over a hemisphere. Proc. R. Soc. Lond. A 232, 184-195.

Hinch, E. J. 1991 Perturbation Methods. Cambridge University Press.

Hopfinger, E. J., Flór, J.-B., Chomaz, J.-M. \& Bonneton, P. 1991 Internal waves generated by a moving sphere and its wake in a stratified fluid. Exps. Fluids 11, 255-261.

Hunt, J. C. R., Feng, Y., Linden, P. F., Greenslade, M. D. \& Mobbs, S. D. 1997 Low-Froudenumber stable flows past mountains. Nuovo Cim. C 20, 261-272.

Hunt, J. C. R. \& RichardS, K. J. 1984 Stratified airflow over one or two hills. Boundary-Layer Met. 30, 223-259. 
Hunt, J. C. R., Richards, K. J. \& Brighton, P. W. M. 1988 Stably stratified shear flow over low hills. Q. J. R. Met. Soc. 114, 859-886.

Hunt, J. C. R. \& SNYDER, W. H. 1980 Experiments on stably and neutrally stratified flow over a model three-dimensional hill. J. Fluid Mech. 96, 671-704.

Huppert, H. E. \& Miles, J. W. 1969 Lee waves in a stratified flow. Part 3. Semi-elliptical obstacle. J. Fluid Mech. 35, 481-496.

JACKSON, J. D. 1999 Classical Electrodynamics, 3rd edn. Wiley.

JANOwITZ, G. S. 1973 Unbounded stratified flow over a vertical barrier. J. Fluid Mech. 58, 375-388.

JANOwitZ, G. S. 1984 Lee waves in three-dimensional stratified flow. J. Fluid Mech. 148, 97-108.

Kadri, Y., Bonneton, P., Chomaz, J.-M. \& Perrier, M. 1996 Stratified flow over three-dimensional topography. Dyn. Atmos. Oceans 23, 321-334.

Kantzios, Y. D. \& Akylas, T. R. 1993 An asymptotic theory of nonlinear stratified flow of large depth over topography. Proc. R. Soc. Lond. A 440, 639-653.

Kozhevnikov, V. N. 1963 A single nonlinear problem of the orographic disturbance of stratified air flow. Bull. (Izv.) Acad. Sci. USSR Geophys. Ser., 675-680. [Transl. from Izv. Akad. Nauk SSSR Ser. Geofiz., 1108-1116.]

Kozhevnikov, V. N. 1968 Orographic perturbations in the two-dimensional stationary problem. Izv. Atmos. Ocean. Phys. 4, 16-27. [Transl. from Izv. Akad. Nauk SSSR Fiz. Atmos. Okeana 4, 33-52.]

Krishna, D. V. 1968 Unsteady stratified flow past a cylinder. Zastosow. Mat. 9, 417-427.

Landau, L. D. \& Lifshitz, E. M. 1987 Fluid Mechanics, 2nd edn. Butterworth-Heinemann.

Landau, L. D., Lifshitz, E. M. \& PitaevskiI, L. P. 1984 Electrodynamics of Continuous Media, 2nd edn. Butterworth-Heinemann.

Laprise, R. \& Peltier, W. R. 1989 On the structural characteristics of steady finite-amplitude mountain waves over bell-shaped topography. J. Atmos. Sci. 46, 586-595.

Lighthill, M. J. 1967 On waves generated in dispersive systems by travelling forcing effects, with applications to the dynamics of rotating fluids. J. Fluid Mech. 27, 725-752.

LighthiLl, J. 1978 Waves in Fluids. Cambridge University Press.

Lighthill, J. 1986 An Informal Introduction to Theoretical Fluid Mechanics. Oxford University Press.

Lilly, D. K. \& KLemP, J. B. 1979 The effects of terrain shape on nonlinear hydrostatic mountain waves. J. Fluid Mech. 95, 241-261.

Lin, Q., Boyer, D. L. \& Fernando, H. J. S. 1993 Internal waves generated by the turbulent wake of a sphere. Exps. Fluids 15, 147-154.

Lin, Q., Lindberg, W. R., Boyer, D. L. \& Fernando, H. J. S. 1992 Stratified flow past a sphere. J. Fluid Mech. 240, 315-354.

LofQuist, K. E. B. \& Purtell, L. P. 1984 Drag on a sphere moving horizontally through a stratified liquid. J. Fluid Mech. 148, 271-284.

LoNG, R. R. 1953 Some aspects of the flow of stratified fluids. I. A theoretical investigation. Tellus $\mathbf{5}, 42-58$.

MacKinnon, R. F., Mulley, R. \& Warren, F. W. G. 1969 Some calculations of gravity wave resistance in an inviscid stratified fluid. J. Fluid Mech. 38, 61-73.

Makarov, S. A. \& Chashechkin, Yu. D. 1981 Apparent internal waves in a fluid with exponential density distribution. J. Appl. Mech. Tech. Phys. 22, 772-779. [Transl. from Zh. Prikl. Mekh. Tekh. Fiz. (6), 47-54.]

Makarov, S. A. \& Chashechkin, Yu. D. 1982 Coupled internal waves in a viscous incompressible fluid. Izv. Atmos. Ocean. Phys. 18, 758-764. [Transl. from Izv. Akad. Nauk SSSR Fiz. Atmos. Okeana 18, 986-994.]

MAson, P. J. 1977 Forces on spheres moving horizontally in a rotating stratified fluid. Geophys. Astrophys. Fluid Dyn. 8, 137-154.

Mellin, R. H. 1910 Abriß einer einheitlichen Theorie der Gamma- und der hypergeometrischen Funktionen. Math. Annln 68, 305-337.

MiLes, J. W. 1968 Lee waves in a stratified flow. Part 1. Thin barrier. J. Fluid Mech. 32, 549-567.

MiLes, J. W. 1969 Waves and wave drag in stratified flows. In Proc. XIIth Intl Congress of Applied Mechanics (ed. M. Hétényi \& W. G. Vincenti), pp. 50-76. Springer.

MiLEs, J. W. 1971 Internal waves generated by a horizontally moving source. Geophys. Fluid Dyn. 2, 63-87. 
Miles, J. W. \& Huppert, H. E. 1968 Lee waves in a stratified flow. Part 2. Semi-circular obstacle. J. Fluid Mech. 33, 803-814.

Miles, J. W. \& Huppert, H. E. 1969 Lee waves in a stratified flow. Part 4. Perturbation approximations. J. Fluid Mech. 35, 497-525.

Miranda, P. M. A. \& James, I. N. 1992 Non-linear three-dimensional effects on gravity-wave drag: Splitting flow and breaking waves. Q. J. R. Met. Soc. 118, 1057-1081.

Murdock, J. W. 1977 The near-field disturbance created by a body in a stratified medium with a free surface. Trans. ASME E: J. Appl. Mech. 44, 534-540.

Newley, T. M. J., Pearson, H. J. \& Hunt, J. C. R. 1991 Stably stratified rotating flow through a group of obstacles. Geophys. Astrophys. Fluid Dyn. 58, 147-171.

PALIERNE, J. F. 1999 On the motion of rigid bodies in incompressible inviscid fluids of inhomogeneous density. J. Fluid Mech. 393, 89-98.

Paris, R. B. \& Kaminski, D. 2001 Asymptotics and Mellin-Barnes Integrals. Cambridge University Press.

Peat, K. S. \& Stevenson, T. N. 1975 Internal waves around a body moving in a compressible density-stratified fluid. J. Fluid Mech. 70, 673-688.

Phillips, D. S. 1984 Analytical surface pressure and drag for linear hydrostatic flow over threedimensional elliptical mountains. J. Atmos. Sci. 41, 1073-1084.

Prasad, D., Ramirez, J. \& Akylas, T. R. 1996 Stability of stratified flow of large depth over finite-amplitude topography. J. Fluid Mech. 320, 369-394.

Rottman, J. W., Broutman, D., Spedding, G. \& Meunier, P. 2004 The internal wave field generated by the body and wake of a horizontally moving sphere in a stratified fluid. In Proc. 15th Australasian Fluid Mechanics Conf. (ed. M. Behnia, W. Lin \& G. D. McBain), pap. AFMC00131. University of Sydney. Available in electronic form at the URL http://www.aeromech.usyd.edu.au/15afmc/proceedings/.

Rotunno, R., Grubiší́, V. \& Smolarkiewicz, P. K. 1999 Vorticity and potential vorticity in mountain wakes. J. Atmos. Sci. 56, 2796-2810.

Rotunno, R. \& Smolarkiewicz, P. K. 1991 Further results on lee vortices in low-Froude number flow. J. Atmos. Sci. 48, 2204-2211.

Rowe, R. D., Benjamin, S. F., Chung, K. P., Havlena, J. J. \& Lee, C. Z. 1981 Field studies of stable air flow over and around a ridge. Atmos. Environ. 16, 643-653.

Ryan, W. \& LAMB, B. 1984 Determination of dividing streamline heights and Froude numbers for predicting plume transport in complex terrain. J. Air Pollut. Control Ass. 31, 152-155.

Sarma, L. V. K. V. \& Krishna, D. V. 1972 Motion of a sphere in a stratified fluid. Zastosow. Mat. 13, 123-130.

SCase, M. M. \& Dalziel, S. B. 2004 Internal wave fields and drag generated by a translating body in a stratified fluid. J. Fluid Mech. 498, 289-313.

SCHÄR, C. \& DurRan, D. R. 1997 Vortex formation and vortex shedding in continuously stratified flows past isolated topography. J. Atmos. Sci. 54, 534-554.

SCORER, R. S. 1956 Airflow over an isolated hill. Q. J. R. Met. Soc. 82, 75-81.

ShePpard, P. A. 1956 Airflow over mountains. Q. J. R. Met. Soc. 82, 528-529.

ShishKinA, O. D. 1996 Comparison of the drag coefficients of bodies moving in liquids with various stratification profiles. Fluid Dyn. 31, 484-489. [Transl. from Izv. Akad. Nauk Mekh. Zhidk. Gaza (4), 4-11.]

Sмith, R. B. 1980 Linear theory of stratified hydrostatic flow past an isolated mountain. Tellus 32, 348-364.

Sмith, R. B. 1988 Linear theory of stratified flow past an isolated mountain in isosteric coordinates. J. Atmos. Sci. 45, 3889-3896.

SMItH, R. B. 1989a Mountain-induced stagnation points in hydrostatic flow. Tellus A 41, 270-274.

SMIth, R. B. $1989 b$ Hydrostatic airflow over mountains. Adv. Geophys. 31, 1-41.

SMith, R. B. \& GRøNÅs, S. 1993 Stagnation points and bifurcation in 3-D mountain airflow. Tellus A 45, 28-43.

Smolarkiewicz, P. K. \& Rotunno, R. 1989 Low Froude number flow past three-dimensional obstacles. Part I: Baroclinically generated lee vortices. J. Atmos. Sci. 46, 1154-1164.

Smolarkiewicz, P. K. \& Rotunno, R. 1990 Low Froude number flow past three-dimensional obstacles. Part II: Upwind flow reversal zone. J. Atmos. Sci. 47, 1498-1511. 
Snyder, W. H., Thompson, R. S., Eskridge, R. E., Lawson, R. E., Castro, I. P., Lee, J. T., Hunt, J. C. R. \& OGawa, Y. 1985 The structure of strongly stratified flow over hills: dividingstreamline concept. J. Fluid Mech. 152, 249-288.

Spangler, T. C. 1987 Comparison of actual dividing-streamline heights to height predictions using the Froude number. J. Clim. Appl. Met. 26, 204-207.

Sturova, I. V. 1974 Wave motions produced in a stratified liquid from flow past a submerged body. J. Appl. Mech. Tech. Phys. 15, 796-805. [Transl. from Zh. Prikl. Mekh. Tekh. Fiz. (6), 80-91.]

STurova, I. V. 1978 Internal waves generated by local disturbances in a linearly stratified liquid of finite depth. J. Appl. Mech. Tech. Phys. 19, 330-336. [Transl. from Zh. Prikl. Mekh. Tekh. Fiz. (3), 61-69.]

Suzuki, M. \& Kuwahara, K. 1992 Stratified flow past a bell-shaped hill. Fluid Dyn. Res. 9, 1-18.

Sykes, R. I. 1978 Stratification effects in boundary layer flow over hills. Proc. R. Soc. Lond. A 361, 225-243.

Sysoeva, E. YA. \& Chashechin, Yu. D. 1986 Vortex structure of a wake behind a sphere in a stratified fluid. J. Appl. Mech. Tech. Phys. 27, 190-196. [Transl. from Zh. Prikl. Mekh. Tekh. Fiz. (2), 40-46.]

Sysoeva, E. Ya. \& Chashechin, Yu. D. 1988 Spatial structure of a wake behind a sphere in a stratified liquid. J. Appl. Mech. Tech. Phys. 29, 655-660. [Transl. from Zh. Prikl. Mekh. Tekh. Fiz. (5), 59-65.]

Sysoeva, E. Ya. \& Chashechin, Yu. D. 1991 Vortex systems in the stratified wake of a sphere. Fluid Dyn. 26, 544-551. [Transl. from Izv. Akad. Nauk SSSR Mekh. Zhidk. Gaza (4), 82-90.]

UMeKi, M. \& Kambe, T. 1989 Stream patterns of an isothermal atmosphere over an isolated mountain. Fluid Dyn. Res. 5, 91-109.

Vladimirov, V. A. \& IL'In, K. I. 1991 Slow motions of a solid in a continuously stratified fluid. J. Appl. Mech. Tech. Phys. 32, 194-200. [Transl. from Zh. Prikl. Mekh. Tekh. Fiz. (2), 55-60.]

Vorsin, B. 1991a Rayonnement des ondes internes de gravité. Application aux corps en mouvement. Ph.D. thesis, Université Pierre et Marie Curie, Paris.

VoIsin, B. $1991 b$ Internal wave generation in uniformly stratified fluids. Part 1. Green's function and point sources. J. Fluid Mech. 231, 439-480.

VoIsIN, B. 1994 Internal wave generation in uniformly stratified fluids. Part 2. Moving point sources. J. Fluid Mech. 261, 333-374.

VoIsIN, B. 1995 Internal wave generation by turbulent wakes. In Mixing in Geophysical Flows (ed. J. M. Redondo \& O. Métais), pp. 291-301. CIMNE.

Vorsin, B. 2003 Limit states of internal wave beams. J. Fluid Mech. 496, 243-293.

Vosper, S. B. 2000 Three-dimensional numerical simulations of strongly stratified flow past conical orography. J. Atmos. Sci. 57, 3716-3739.

Vosper, S. B., Castro, I. P., Snyder, W. H. \& Mobbs, S. D. 1999 Experimental studies of strongly stratified flow past three-dimensional orography. J. Fluid Mech. 390, 223-249.

Warren, F. W. G. 1960 Wave resistance to vertical motion in a stratified fluid. J. Fluid Mech. 7, 209-229.

Watson, G. N. 1944 A Treatise on the Theory of Bessel Functions, 2nd edn. Cambridge University Press.

Wu, T. Y.-T. 1965 Three-dimensional internal gravity waves in a stratified free-surface flow. Z. Angew. Math. Mech. Sonderh. 45, T194-T195.

Wurtele, M. G. 1957 The three-dimensional lee wave. Beitr. Phys. Atmos. 29, 242-252.

Wurtele, M. G., Sharman, R. D. \& Datta, A. 1996 Atmospheric lee waves. Annu. Rev. Fluid Mech. 28, 429-476.

YiH, C.-S. 1967 Equations governing steady three-dimensional large-amplitude motion of a stratified fluid. J. Fluid Mech. 29, 539-544.

Zeytounian, R. Kн. 1969 Phénomènes d'ondes dans les écoulements stationnaires d'un fluide stratifié non visqueux. Première partie. Modèles théoriques. J. Méc. 8, 239-263. 